\title{
Mechanochemical modeling of neutrophil migration based on four signaling layers, integrin dynamics, and substrate stiffness
}

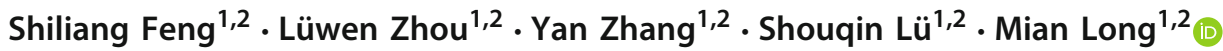

Received: 21 March 2018 / Accepted: 24 June 2018

c) Springer-Verlag GmbH Germany, part of Springer Nature 2018

\begin{abstract}
Directional neutrophil migration during human immune responses is a highly coordinated process regulated by both biochemical and biomechanical environments. In this paper, we developed an integrative mathematical model of neutrophil migration using a lattice Boltzmann-particle method built in-house to solve the moving boundary problem with spatiotemporal regulation of biochemical components. The mechanical features of the cell cortex are modeled by a series of spring-connected nodes representing discrete cell-substrate adhesive sites. The intracellular signaling cascades responsible for cytoskeletal remodeling [e.g., small GTPases, phosphoinositide-3-kinase (PI3K), and phosphatase and tensin homolog] are built based on our previous four-layered signaling model centered on the bidirectional molecular transport mechanism and implemented as reaction-diffusion equations. Focal adhesion dynamics are determined by force-dependent integrin-ligand binding kinetics and integrin recycling and are thus integrated with cell motion. Using numerical simulations, the model reproduces the major features of cell migration in response to uniform and gradient biochemical stimuli based on the quantitative spatiotemporal regulation of signaling molecules, which agree with experimental observations. The existence of multiple types of integrins with different binding kinetics could act as an adaptation mechanism for substrate stiffness. Moreover, cells can perform reversal, U-turn, or lock-on behaviors depending on the steepness of the reversal biochemical signals received. Finally, this model is also applied to predict the responses of mutants in which PTEN is overexpressed or disrupted.
\end{abstract}

Keywords Chemotaxis $\cdot$ Cytoskeletal remodeling $\cdot$ Mathematical model $\cdot$ Biochemical $\cdot$ Biomechanical

\section{Introduction}

Understanding the migration mechanism of neutrophils is a crucial issue in immune responses (Kolaczkowska and Kubes 2013). Generally, as a fast moving amoeboid-type cell, a neutrophil migrates by invoking a complexly controlled and

Shiliang Feng and Lüwen Zhou have contributed equally to this work.

Electronic supplementary material The online version of this article (https://doi.org/10.1007/s10237-018-1047-2) contains supplementary material, which is available to authorized users.

$\triangle$ Mian Long

mlong@imech.ac.cn

1 Center for Biomechanics and Bioengineering, Key Laboratory of Microgravity (National Microgravity Laboratory), and Beijing Key Laboratory of Engineered Construction and Mechanobiology, Institute of Mechanics, Chinese Academy of Sciences, Beijing, China

2 School of Engineering Science, University of Chinese Academy of Sciences, Beijing, China integrated sequence of events, protrusion, adhesion, and contraction (Bagorda and Parent 2008; Fenteany and Glogauer 2004). Initially, the assembly of cross-linked actin filaments drives the protrusion of a thin sheet-like structure, called the lamellipod, at the leading edge (Fenteany and Glogauer 2004; Ridley et al. 2003). Next, the cell strengthens its adhesion to the extracellular matrix (ECM) at the leading edge and weakens its adhesion at the rear edge (Nagano et al. 2012; Yang et al. 2017). Finally, myosin molecular motors bind the bundled actin filaments and exert contractile stress to pull the cell rear forward (Rottner and Stradal 2011; Shin et al. 2010). Under in vivo conditions, the direction of neutrophil migration is largely determined by the gradient of pathogen-associated biochemical signals (Jin 2013), while the cell morphology and migration velocity are dramatically affected by the mechanical properties of the substrate (Zaman et al. 2006).

Interestingly, even in the absence of biochemical guidance, an adhesive neutrophil is not static, as it constantly extends and retracts protrusions, suggesting that its cytoskele- 
tal remodeling process could be excited by internal signaling noise (Swaney et al. 2010). Recently, measurements on endogenous Rac activation (Lin et al. 2012) and exogenous $\mathrm{PIP}_{3}$ delivery (Weiner et al. 2002) via bypassing upstream cascades confirmed that the polarity of the cytoskeleton is largely built upon positive/negative feedback loops mediated by small Rho GTPases (including Cdc42, Rac, and RhoA) and membrane lipids (including $\mathrm{PIP}_{2}$ and $\mathrm{PIP}_{3}$, hereafter defined as PIs) (Weiner 2002). If a neutrophil is stimulated by a graded biochemical signaling field, the GPCR-controlled, cytoskeleton-independent pathway (involving the feedback cycle of PI3K-Ras) is initiated and serves as a compass mechanism for controlling the directional regulation of Rho GTPases (Damoulakis et al. 2014), and thus, cytoskeletal remodeling (Sasaki et al. 2007).

When a neutrophil moves, complex mechano-sensing signaling pathways are activated due to adhesive bond stretching, thereby generating mechanical-chemical feedback from the substrate to the cell (Giannone and Sheetz 2006). Among various molecular bases (i.e., paxillin, FAK, vinculin, talin, and integrin) (Giannone and Sheetz 2006), integrins are the most important molecules underlying the adhesive mechanosensing phenomenon (Shibata et al. 2013). Integrins serve as both the molecular hub for localizing adhesive molecular signaling (Scales and Parsons 2011) and as the cell-substrate physical connection for detecting mechanical properties of the substrate (Schaefer and Hordijk 2015). Using multiple techniques, such as atomic force microscopy, magnetic tweezers, and optical tweezers, the binding kinetics and forced dissociation of distinct integrin-ligand bonds have been isolated and identified (Sako et al. 2012; EloseguiArtola et al. 2014; Liang et al. 2008). For example, the binding of $\beta_{2}$-integrin member LFA-1 to the intercellular adhesion molecule 1 (ICAM-1) ligand yields a smaller dissociation rate and a larger association rate than that of another member, Mac-1, to ICAM-1 (Li et al. 2013).

Numerous mathematical/computational models of eukaryotic cell mobility have previously been developed (Iglesias and Devreotes 2008; Karsenti 2008; Danuser et al. 2013). While these models provide qualitative and/or quantitative insights into the distinct features of cell motility based on specific simplifications, the coupling between biochemical and biomechanical environments to regulate cytoskeletal remodeling and focal adhesion dynamics remains oversimplified. For example, previous models typically treated the cytoskeleton as a mechanical framework consisting of a mesh of discrete nodes connected by elastic springs and viscous dashpots (Sarvestani and Jabbari 2009; Dokukina and Gracheva 2010; Bottino et al. 2002). While solving the force-balance equations at each node reasonably agrees with the experimentally measured cell moving velocity, these models require that the cell be polarized (i.e., the cell has a well-defined front and rear). Meanwhile, the cell boundary alteration induced by a regulatory signaling network inside the cell is described by a conceptual polarization mechanism (Wolgemuth et al. 2011). Generally, biomarkers for the cell protrusive front are elaborately defined, but those for the contractive rear are unelucidated. A more recent model on integrative cell migration (Kim et al. 2012) incorporates focal adhesion dynamics and cytoskeletal remodeling but does not include the biophysical properties of ECM and therefore fails to capture the spatiotemporally coordinated events of intracellular signaling dynamics.

Here, we developed an integrative neutrophil migration model that couples the cellular focal adhesion dynamics and intracellular signaling responsible for cytoskeletal remodeling and morphological change. We extended our previously proposed signaling models (Feng and Zhu 2014; Feng et al. 2018) in two aspects. First, a focal adhesion dynamics module dominated by force-dependent integrinligand binding kinetics and integrin recycling was incorporated to describe the mechano-sensing machinery. Second, a viscoelastic mechanical model was introduced to simulate cytoskeleton-mediated cell deformation and movement. An in-house-developed lattice Boltzmann-particle (LBP) method was utilized to treat the moving boundary problem (MBP) efficiently, wherein the particle method was adopted to reassess the mechanical boundary condition and the lattice Boltzmann (LB) method for solving the reaction-diffusion system on the newly born cell domain. Additionally, a simple Monte Carlo (MC) method was embedded to treat the stochastic reaction source terms. This model can account for the spontaneous, directional, and turning dynamics of cell migration in response to uniform, graded, and reversal biochemical stimuli, respectively. It generates mutant migration via the modification of effector molecular concentrations. Moreover, varying integrin-ligand binding kinetics can serve as an adaptation mechanism to varied substrate stiffness.

\section{Materials and methods}

In this section, we summarize the theoretical modeling with simplified assumptions (for more detailed model assumptions, see the Supporting Information). This model consists of three modules. The first two modules describe the signaling cascades responsible for cytoskeletal remodeling and focal adhesion dynamics, respectively, and are governed by coupled reaction-diffusion equations. The third module denotes cell mechanics and motility and is governed by force-balance equations.

\subsection{Module for four signaling layers}

This module involves four layers (Fig. 1a), (I) signal reception, (II) initial signaling processing, (III) small Rho GTPase 


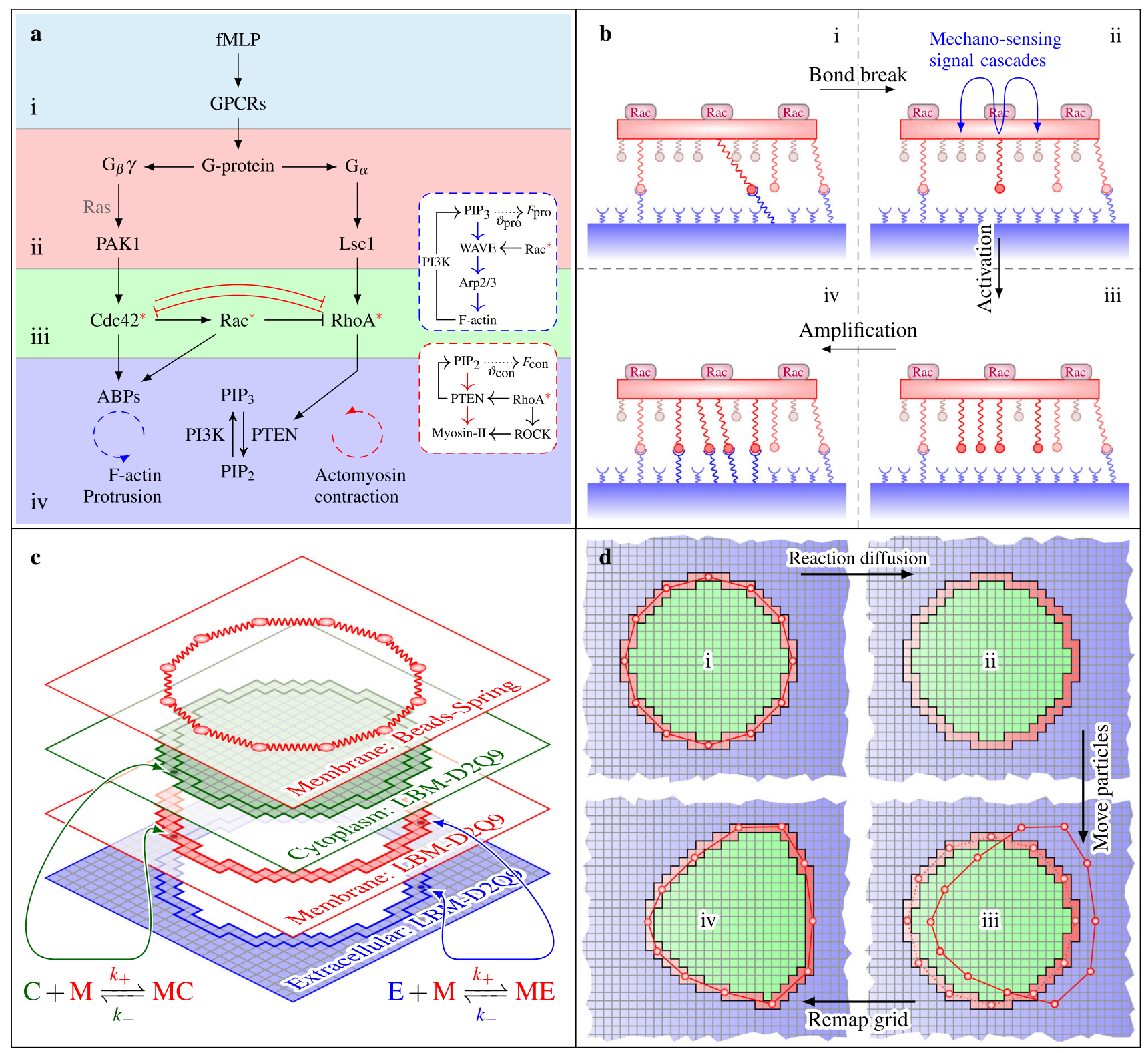

Fig. 1 A working computational model for neutrophil chemotaxis. a Four-layered signaling cascades responsible for cytoskeletal remodeling. (i) Signal reception layer: A cell detects soluble factor fMLP by G protein-coupled receptors (GPCRs). (ii) Initial signal processing layer: G protein dissociates into $\mathrm{G}_{\alpha}$ and $\mathrm{G}_{\beta} \gamma$ subunits, which triggers a fast activation and a slow inhibition response. (iii) Small Rho GTPase regulation layer: Spatial regulation of Rho GTPase members (Rac, Cdc42, and RhoA) is achieved by the localization of GEFs (i.e., PAK1 and Lsc1) and antagonistic effects among the members (*denotes their active forms). (iv) Bidirectional molecular transport layer: Spatial effects generated from Rho GTPase-PI feedback loops manipulate bidirectional cytoskeletal remodeling. The curved blue and red arrows indicate the feedback loops for generating protrusive and contractive forces, respectively. The inserts show the molecular details that are treated implicitly in our model. The arrows colored blue and red in the inserts represent the pathways for providing membrane binding sites, indicating the applicability of the mass conservation law. b Schematic of the focal

adhesion dynamics module. Inactive, unbound active, and bound integrins are indicated by blue, green, and red springs, respectively. The dense springs represent the stiff matrix. Four steps in integrin regulation are depicted: (i) integrin binding; (ii) once a sufficient stretch force is exerted on the bond, the bond can serve as a source point for initiating mechano-sensing signaling cascades; (iii) the local strength of intracellular integrin activation is mediated by the active Rac concentration; and (iv) ultimate amplification. c LBP method implementation of the model. Mechanically, the cell is modeled by a series of spring-connected nodes (top layer). The entire computational domain is discretized by the D2Q9 lattice model. Lattices colored red, green, and blue are specified as the membrane, cytosol, and extracellular environment, respectively. Molecular translocation described by reaction equations occurs at the shared lattices. d Simulation procedures. (i) Solving reaction-diffusion equations. (ii) Specifying nodal forces based upon the lattice concentration. (iii) Movement. (iv) Lattice-particle remapping (see Fig. S1 for more details) 
regulation, and (IV) bidirectional molecular transport. Details on the overall modeling strategy and justifying the assumed cross talk among these modules have been previously described (Herant et al. 2005) and are briefly discussed below.

(I) Signal reception Diffusion and diminution of a chemoattractant, i.e., fMLP, are modeled by the reactiondiffusion equation with corresponding initial and boundary conditions

$\frac{\partial S}{\partial t}=D_{f} \nabla^{2} S-K S$

$S(x, y, t)=S_{0}, \quad$ for $\quad x \in\left[x_{1}, x_{2}\right], y \in\left[y_{1}, y_{2}\right]$,

$S(x, y, 0)=0, \quad$ for $\quad x \notin\left[x_{1}, x_{2}\right], y \notin\left[y_{1}, y_{2}\right]$,

$S(X, Y, t)=0$.

Here, $S, D_{f}$, and $K$ are the concentration, diffusion coefficient, and delay rate of fMLP, respectively. $x \in$ $\left[x_{1}, x_{2}\right]$ and $y \in\left[y_{1}, y_{2}\right]$ define the domain of the source point wherein the concentration of fMLP equals $S_{0}$. $X$ and $Y$ represent the boundary of the fMLP field. The binding kinetics of fMLP molecules (as ligands) to GPCRs (as receptors) are described by the following reaction equation:

$\mathrm{R}+\mathrm{L} \underset{k_{-}}{\stackrel{k_{+}}{\rightleftharpoons}} \mathrm{RL}$,

where $\mathrm{R}, \mathrm{L}, \mathrm{RL}, k_{+}$, and $k_{-}$represent the receptor, ligand, bond, association rate, and dissociation rate, respectively.

(II) Initial signal processing A balance-inactivation mechanism is used to mimic the initial signaling process (Levine et al. 2006). This mechanism involves three interacting steps: (i) The local receptor occupancy level ([RL]) drives the production of membrane-bound species $A$ and cytosolic species $I$ at equal rates, $k_{\mathrm{S}}$; (ii) the cytosolic species diffuses inside the cell and attaches itself to the membrane at a rate $k_{\mathrm{I}}$ and becomes the membrane-anchored species $I_{\mathrm{m}}$; and (iii) both species $A$ and $I_{\mathrm{m}}$ inactivate each other with a rate $k_{\mathrm{i}}$, and $A$ and $I_{\mathrm{m}}$ spontaneously degrade at rates $\delta_{\mathrm{A}}$ and $\delta_{\mathrm{I}}$, respectively. The system equations can be written as

$$
\begin{aligned}
\frac{\partial A}{\partial t} & =D_{\mathrm{m}} \nabla^{2} A+k_{\mathrm{s}}[\mathrm{RL}]-\delta_{\mathrm{A}} A-k_{\mathrm{i}} A I_{\mathrm{m}} \\
\frac{\partial I_{\mathrm{m}}}{\partial t} & =D_{\mathrm{m}} \nabla^{2} I_{\mathrm{m}}+k_{\mathrm{I}} I-\delta_{\mathrm{I}} I_{\mathrm{m}}-k_{\mathrm{i}} A I_{\mathrm{m}}, \\
\frac{\partial I}{\partial t} & =D_{\mathrm{c}} \nabla^{2} I
\end{aligned}
$$

with the boundary condition

$D_{\mathrm{c}} \frac{\partial I}{\partial n}=k_{\mathrm{s}}[\mathrm{RL}]-k_{\mathrm{I}} I$.

The translocating behaviors of PAK1 and Lsc1 from the cytosol to the membrane (like those of PI3K and PTEN) are described by the binding reaction equation [Eq. (3)], where PAK1 and Lsc1 act as ligands, while $A$ and $I_{\mathrm{m}}$ function as receptors. The spatiotemporal evolution of cytosolic components obeys the following standard diffusion equation:

$$
\frac{\partial E_{\mathrm{c}}}{\partial t}=D_{\mathrm{c}} \nabla^{2} E_{\mathrm{c}}
$$

with the boundary condition

$D_{\mathrm{c}} \frac{\partial E_{\mathrm{c}}}{\partial n}=-k_{+} S_{\mathrm{m}} E_{\mathrm{m}}+k_{-} E_{\mathrm{m}}$

where $E_{\mathrm{c}}=E_{\mathrm{PAK} 1}, E_{\mathrm{Lsc} 1}, E_{\mathrm{PI} 3 \mathrm{~K}}$, and $E_{\mathrm{PTEN}}$ represents the cytosolic concentrations of PAK1, Lsc1, PI3K, and PTEN, respectively, $S_{\mathrm{m}}$ denotes the corresponding receptor concentration, and $E_{\mathrm{m}}$ represents the corresponding membrane-bound form.

(III) Small Rho GTPase regulation Each Rho GTPase member may cycle between active membrane-bound and inactive cytosolic forms (Lin et al. 2012). The spatiotemporal evolutions of active Rho GTPases are described as follows:

$\frac{\partial G}{\partial t}=D_{\mathrm{m}} \nabla^{2} G+P_{\mathrm{G}}(R, C, \rho)\left(\frac{G_{\mathrm{i}}}{G_{\mathrm{tot}}}\right)-\omega_{\mathrm{G}} G$,

where $G=R, C$, and $\rho$ represent the active (membranebound) forms of Rac, $\mathrm{Cdc} 42$, and RhoA, respectively. $G_{\text {tot }}=R_{\text {tot }}, C_{\text {tot }}$, and $\rho_{\text {tot }}$ are the total amounts of Rac, Cdc42, and RhoA, respectively. $G_{\mathrm{i}}=R_{\mathrm{i}}, C_{\mathrm{i}}$, and $\rho_{\mathrm{i}}$ are the total amounts of the respective inactive (cytosol) forms of Rac, Cdc42, and RhoA that are calculated by the conservation law. $P_{\mathrm{G}}$ is the activation term and is expressed as

$P_{\mathrm{R}}=I_{\mathrm{R}}+\alpha C, P_{\mathrm{C}}=I_{\mathrm{C}}+\beta E_{\mathrm{C}}, P_{\rho}=I_{\rho}+\tau E_{\rho}$.

Here, $I_{\mathrm{R}}, I_{\mathrm{C}}$, and $I_{\rho}$ are the baseline activation rates. $\alpha$ determines the rate of $\mathrm{Cdc} 42$-enhanced Rac activation, and $\beta$ and $\tau$ are the rates of the guanine exchange factor (GEF)-mediated activation of $\mathrm{Cdc} 42$ and RhoA, respectively, $\omega_{\mathrm{G}}$ is the inactivation term and is expressed as

$\omega_{\mathrm{R}}=\delta_{\mathrm{R}}+\gamma \rho, \omega_{\mathrm{C}}=\delta_{\mathrm{C}}+\varepsilon \rho, \omega_{\rho}=\delta_{\rho}+\varepsilon C$.

Here, $\delta_{\mathrm{R}}, \delta_{\mathrm{C}}$, and $\delta_{\rho}$ are the GAP-mediated baseline inactivation rates. $\gamma$ is the rate of RhoA-mediated Rac 
inactivation, and $\varepsilon$ represents the mutual inactivation rate of $\mathrm{Cdc} 42$ and RhoA.

(IV) Bidirectional molecular transport The spatiotemporal regulation of $\mathrm{PIP}_{3}\left(P_{3}\right)$ and $\mathrm{PIP}_{2}\left(P_{2}\right)$ forms the core of the bidirectional molecular transport mechanism, which is described by the following equations:

$$
\begin{gathered}
\frac{\partial P_{3}}{\partial t}=D_{\mathrm{m}} \nabla^{2} P_{3}+k_{\mathrm{cat}}^{\mathrm{PI} 3 \mathrm{~K}}\left(\frac{H_{\mathrm{m}} P_{2} R}{k_{\mathrm{M}}^{\mathrm{P} 3 \mathrm{~K}}+P_{2}}\right) \\
-k_{\mathrm{cat}}^{\mathrm{PTEN}}\left(\frac{T_{\mathrm{m}} P_{3} \rho}{k_{\mathrm{M}}^{\mathrm{PTEN}}+P_{3}}\right), \\
\frac{\partial P_{2}}{\partial t}=D_{\mathrm{m}} \nabla^{2} P_{2}-k_{\mathrm{cat}}^{\mathrm{PI} 3 \mathrm{~K}}\left(\frac{H_{\mathrm{m}} P_{2} R}{k_{\mathrm{M}}^{\mathrm{PIK}}+P_{2}}\right) \\
+k_{\mathrm{cat}}^{\mathrm{PTEN}}\left(\frac{T_{\mathrm{m}} P_{3} \rho}{k_{\mathrm{M}}^{\mathrm{PTEN}}+P_{3}}\right) .
\end{gathered}
$$

where, $\tilde{R}=\min \left\{R / R_{\max }, 1\right\}$ and $\tilde{\rho}=\min \left\{\rho / \rho_{\max }, 1\right\}$. In Eq. (13), the first term on the right-hand side accounts for $\mathrm{PIP}_{3}$ diffusion, the second term accounts for $\mathrm{PIP}_{3}$ production due to membrane-bound $\mathrm{PI} 3 \mathrm{~K}$ $\left(H_{\mathrm{m}}\right)$ acting on $\mathrm{PIP}_{2}$, and the third term accounts for $\mathrm{PIP}_{3}$ diminution due to membrane-bound PTEN acting on $\mathrm{PIP}_{3}$. The parameters $k_{\mathrm{cat}}^{\mathrm{PI} 3 \mathrm{~K}}\left(k_{\mathrm{cat}}^{\mathrm{PTEN}}\right)$ and $k_{\mathrm{M}}^{\mathrm{PI} 3 \mathrm{~K}}$ $\left(k_{\mathrm{M}}^{\mathrm{PTEN}}\right)$ are based on the steady state levels of PIs. $\tilde{R}$ $(\tilde{\rho})$ is the normalized factor reflecting the effect of Rac (Rho) activity on PI3K (PTEN) activation, and $R_{\max }$ $\left(\rho_{\max }\right)$ acts as a constant of Rac (RhoA) activity. If $\tilde{R}(\tilde{\rho})$ is greater than $R_{\max }\left(\rho_{\max }\right)$, the activity of Rac (RhoA) is no longer a limiting factor for PI3K (PTEN) activation, and $\tilde{R}(\tilde{\rho})$ then equals unity. Equation (14) describes PIP $_{2}$ dynamics. Similarly, the first term on the right accounts for $\mathrm{PIP}_{2}$ diffusion, the second term accounts for $\mathrm{PIP}_{2}$ production from $\mathrm{PIP}_{3}$ via membranebound PTEN $\left(T_{\mathrm{m}}\right)$, and the third term accounts for the reduction of $\mathrm{PIP}_{2}$ into $\mathrm{PIP}_{3}$ via membrane-bound PI3K.

\subsection{Module for focal adhesion dynamics}

Formation of the focal adhesion complex is a stochastic process due to the intrinsic features of binding and unbinding between integrins on the cell membrane and their ligands on the substrate surface. A general one-step multivalent reversible reaction can be written as

$v^{\mathrm{r}} m_{\mathrm{r}}+v^{1} m_{1} \underset{k_{\mathrm{off}}}{\stackrel{k_{\mathrm{on}}}{\rightleftharpoons}} v^{\mathrm{b}} m_{\mathrm{b}}$,

where $m_{\mathrm{r}}, m_{\mathrm{l}}$, and $m_{\mathrm{b}}$ denote integrin, ligand, and integrinligand bonds, respectively, and $v_{\mathrm{r}}, v_{\mathrm{l}}$ and $v_{\mathrm{b}}$ denote the corresponding stoichiometric coefficients $\left(v_{\mathrm{r}}=v_{\mathrm{l}}=v_{\mathrm{b}}=1\right.$ in the current work). $k_{\text {off }}$ and $k_{\text {on }}$ represent the off and on rates, respectively, which are updated after each simulation time interval $\left(\Delta t_{\mathrm{L}}\right) . k_{\mathrm{off}}$ is commonly described by the Bell model (Bell 1978), and $k_{\text {on }}$ is determined by the site density of active integrins. Because the timescale of integrin recycling, i.e., endocytosis or self-delay, is much longer than that of neutrophil polarization, i.e., more than 30 versus $2-3 \mathrm{~min}$ (Böttcher et al. 2012), the total numbers of membrane-bound active and inactive integrins are assumed to be conserved. The total numbers of active and inactive integrins are assumed to be conserved. The transformation of integrins from the inactive state to the active state is determined by the stretch force $\left(f_{q}^{i}\right)$ applied (see Supporting Information). The evolution of inactive $(w)$ and active integrins $\left(w^{*}\right)$ is schematically shown in Fig. 1b and is described by the following reactiondiffusion equations:

$$
\begin{aligned}
\frac{\partial w}{\partial t} & =D_{\mathrm{I}} \nabla^{2} w+\varsigma w^{*}-\rho_{\mathrm{Rac}}\left(\frac{v w}{w_{0}+w}\right), \\
\frac{\partial w^{*}}{\partial t} & =D_{\mathrm{A}} \nabla^{2} w^{*}-\varsigma w^{*}+\rho_{\mathrm{Rac}}\left(\frac{\nu w}{w_{0}+w}\right),
\end{aligned}
$$

where $D_{\mathrm{I}}$ and $D_{\mathrm{A}}$ are the diffusion coefficients for the inactive and active integrins, respectively, $\varsigma$ is the self-delay rate of active integrins, and $w_{0}$ is the concentration of inactive integrins that gives the half maximal conversion rate. $v$ is the source term defined as follows:

$v=\left\{\begin{array}{lll}\nu_{0}+\delta \Psi & \text { if } \quad f_{q}^{i} \geq F_{r} \\ v_{0} & \text { if } \quad f_{q}^{i}<F_{r}\end{array}\right.$

where $\nu_{0}$ is the basal rate of integrin activation, $\delta \Psi$ is the rate of force-mediated integrin activation, and $F_{r}$ is the threshold force. The dynamics of focal adhesion are regulated by chemoattractant concentrations based on the active level of $\operatorname{Rac}\left(\rho_{\text {Rac }}\right)$.

\subsection{Module for cell mechanics}

We use the membrane to model two biological entities, the plasma membrane and the underlying cytoskeleton or cortex. The neutrophil membrane is represented by a discrete springconnected particle circle (with $N$ elastic springs connected at $N$ nodes). The motion of each node $i$ is described by the following force-balance equation:

$m_{i} \frac{\mathrm{d} \mathbf{v}_{i}}{\mathrm{~d} t}=\mathbf{F}_{i}^{\mathrm{elas}}+\mathbf{F}_{i}^{\mathrm{vis}}+\mathbf{F}_{i}^{\mathrm{pro}}+\mathbf{F}_{i}^{\mathrm{con}}+\mathbf{F}_{i}^{\mathrm{drag}}$,

where $\mathbf{v}_{i}$ is the velocity vector, $m_{i}$ is the nodal mass, and $\mathbf{F}_{i}^{\text {elas }}, \mathbf{F}_{i}^{\text {vis }}, \mathbf{F}_{i}^{\text {pro }}, \mathbf{F}_{i}^{\text {con }}$, and $\mathbf{F}_{i}^{\text {drag }}$ are the elastic energy, viscous energy, protrusive, contractive, and drag forces, respectively. A detailed explanation of each term is given as follows: 
- Passive viscoelastic force The elastic energy stored in the springs due to stretching or compression is given by

$$
E_{1}=\frac{1}{2} \sum_{i=1}^{n} K_{1}\left(\frac{l_{i}-l_{0}}{l}\right)^{2}
$$

where $l_{i}$ is the length of the $i$ th spring that is iterated at every step, $l_{0}$ is the relaxed (zero-force) length, and $K_{1}$ is the effective stiffness constant of the spring. The elastic energy stored in the spring due to bending is given by

$$
E_{\mathrm{b}}=\frac{1}{2} \sum_{i=1}^{n} K_{\mathrm{b}} \tan ^{2}\left(\frac{\theta_{i}-\theta_{0}}{2}\right)
$$

where $\theta_{i}$ is the angle between a pair of consecutive springs $(i, i+1)$ that is iterated at every step, $\theta_{0}$ is the relaxed (zero-force) angle, and $K_{\mathrm{b}}$ is the spring constant for bending. In addition, an area constraint is also applied to ensure that the cell area is conserved within $1 \%$ during the simulation. This is implemented via an energetic penalty as follows:

$E_{\mathrm{s}}=\frac{1}{2} K_{\mathrm{s}}\left(\frac{s-s_{0}}{s}\right)^{2}$

where $s$ and $s_{0}$ are the instantaneous and equivalent areas of the cell, respectively, and $K_{\mathrm{S}}$ is the penalty coefficient. The total elastic energy $\left(E_{\mathrm{m}}\right)$ of the cell membrane is the sum of all three types of energy:

$E_{\mathrm{m}}=E_{1}+E_{\mathrm{b}}+E_{\mathrm{s}}$.

The elastic force acting on the membrane particles is then calculated using the principle of the virtual work as follows:

$\mathbf{F}_{i}^{\mathrm{elas}}=-\frac{\partial E_{\mathrm{m}}}{\partial \mathbf{P}_{i}}$

where $\mathbf{P}_{i}$ is the position vector of the $i$ th node. The viscous force is given by

$\mathbf{F}_{i}^{\mathrm{vis}}=-\gamma \mathbf{v}_{i j}$

where $\gamma$ is the viscosity coefficient of the cellular cytoskeleton, and $v_{i j}$ is the relative displacement rate of the neighboring nodes $i$ and $j$.

- Active protrusive and contractive forces Physically, the generation of protrusive and contractive forces can be described by the Brownian ratchet model (Danuser et al. 2013) and the force-velocity relation of the molecular motor (Kim et al. 2012), respectively. Here, we simply assume that the nodal protrusive force $\left(\mathbf{F}_{i}^{\text {pro }}\right)$ is related to the local concentration of $\mathrm{PIP}_{3}\left(P_{3}\right)$, and the nodal contractive force $\left(\mathbf{F}_{i}^{c o n}\right)$ is correlated with that of $\mathrm{PIP}_{2}$ $\left(P_{2}\right)$. The general forms of $\mathbf{F}_{i}^{\text {pro }}$ and $\mathbf{F}_{i}^{\text {con }}$ are given by

$$
\begin{aligned}
\mathbf{F}_{i}^{\mathrm{pro}} & =\frac{v_{\mathrm{pro}} \eta_{i} P_{3}}{2 P_{3 \mathrm{~s}}}\left(l_{i} \hat{\mathbf{n}}_{i}+l_{i+1} \hat{\mathbf{n}}_{i+1}\right), \\
\mathbf{F}_{i}^{\mathrm{con}} & =\frac{-v_{\mathrm{con}} \eta_{i} P_{2}}{2 P_{2 \mathrm{~s}}}\left(l_{i} \hat{\mathbf{n}}_{i}+l_{i+1} \hat{\mathbf{n}}_{i+1}\right),
\end{aligned}
$$

where $v_{\text {pro }}\left(v_{\text {con }}\right)$ is the protrusive (contractive) forceconcentration transfer factor, which is derived from the maximum active force generated by a cell, $P_{3 \mathrm{~s}}\left(P_{2 \mathrm{~s}}\right)$ is the saturation concentration of $\operatorname{PIP}_{3}\left(\mathrm{PIP}_{2}\right)$, and $\hat{\mathbf{n}}_{i}\left(\hat{\mathbf{n}}_{i+1}\right)$ is the outward unit normal vector. $\eta_{i}$ is a normalized factor that reflects the magnitude dependence of $\mathbf{F}_{i}^{\text {pro }}\left(\mathbf{F}_{i}^{\mathrm{con}}\right)$ on the cell-substrate adhesion strength and calculated by the following Langmuir-Hill equation:

$\eta_{i}=\frac{\left(N_{i}^{\mathrm{b}}\right)^{2}}{\left(N_{\mathrm{opt}}\right)^{2}+\left(N_{i}^{\mathrm{b}}\right)^{2}}$.

Here, $N_{\text {opt }}$ is the typical number of closed integrin-ligand bonds (formed only from active integrins) above which the adhesion strength is saturated, and $N_{i}^{\mathrm{b}}$ is the number of closed bonds on the $i$ th node.

- Substrate frictional drag The drag force $\left(F_{i}^{\text {drag }}\right)$ comes from two sources, the viscous drag force and the energy dissipated by the rupture of bonds under the stretch force, which is proportional to the cell velocity $\left(v_{i}\right) . F_{i}^{\mathrm{drag}}$ can be written as

$\mathbf{F}_{i}^{\mathrm{drag}}=\left(\eta_{0}+\eta\right) \mathbf{v}_{i}$

where $\eta_{0}$ is the minimum value of viscosity. Based on the approach proposed in Dokukina and Gracheva (2010), $\eta$ is assumed to be a linearly increasing function of substrate stiffness $\left(k_{\text {sub }}\right)$, i.e., $\eta=c_{\text {vis }} k_{\text {sub }}$, where $c_{\text {vis }}$ is its slope.

\subsection{Numerical simulations}

To investigate mechanical-chemical coupling in cell shape and cell motion, suitable models and computational algorithms are required. Here, an LBP method developed inhouse is applied to solve the MBP (Figs. 1c, d, S1). The solution of the reaction-diffusion system is then coupled to the mechanical model since it appears in the force terms $\left(\mathbf{F}_{i}^{\text {pro }}\right.$ and $\mathbf{F}_{i}^{\text {con }}$ ), and the solution of the force-balance equations thus determines the location of the domain edge. Two time steps, $\Delta t_{\mathrm{L}}$ and $\Delta t_{\mathrm{M}}$, are defined for numerically solving the coupled system, representing time discretization in the diffusion-reaction and force-balance equations, respectively. During one simulation cycle $(T)$, assuming there are 
$\mathrm{m} \mathrm{LB}$ and MC steps and $\mathrm{n}$ particle method steps, $m \Delta t_{\mathrm{L}}=$ $n \Delta t_{\mathrm{M}}=T$, and each time step is sufficiently small to accurately capture the underlying physical phenomenon. A spatial step size of $\Delta x=0.2 \mu \mathrm{m}$ is used, and the number of partials is given by $N=60$. All intracellular variables are uniformly set as the initial condition. Prior to each run, the modeled cell system is preset to undergo a self-evolution for $30 \mathrm{~s}$ (data not shown) such that artificial bias derived from the initial condition setting is largely excluded. Tables S1, S2, and S3 summarize all the parameters used for the simulation. The detailed numerical method is supplied in the Supporting Information.

\section{Results}

\subsection{Modeling spontaneous migration}

A neutrophil placed on a flat substrate with a stiffness of 5 $\mathrm{kPa}$ and an ICAM-1 site density of $30 \mu \mathrm{m}^{-2}$ is considered to represent a typical physiological condition. As shown in Fig. 2a, the trajectories of the cell centroid are totally different in each run, indicating spontaneous cell migration, and the spatiotemporal responses of PIs and integrins in a typical case are presented. Apparently, small Rho GTPase members undergo only self-evolution and are not spatially regulated due to the lack of directional biochemical stimuli. However, their baseline activities appear to provide a limited "driving force" to evoke cytoskeletal remodeling. In fact, acting as effectors, PI3K and PTEN, can translocate from the cytosol to the membrane. After being activated by active Rac and RhoA, respectively, the catalytic effect of PI3K leads to the conversion of $\mathrm{PIP}_{2}$ to $\mathrm{PIP}_{3}$ and vice versa for PTEN. During this process, the stochastic noise generated from effector translocation is amplified locally due to the positive feedback loop of $\mathrm{Rac} \rightarrow \mathrm{PI} 3 \mathrm{~K} \rightleftharpoons \mathrm{PIP}_{3}$, leading to the appearance of $\mathrm{PIP}_{3}$ patches (Fig. 2b, top). Note that one of the $\mathrm{PIP}_{3}$ patches grows at $t \sim 160 \mathrm{~s}$ and becomes gradually dominant. Meanwhile, as $\mathrm{PIP}_{2}$ has the same diffusivity with $\mathrm{PIP}_{3}$ and their total amounts are constant, the pattern of $\mathrm{PIP}_{2}$ regulation mirrors that of $\mathrm{PIP}_{3}$ (Fig. 2b, bottom). Considering that the concentration fields of $\mathrm{PIP}_{3}$ and $\mathrm{PIP}_{2}$ are correlated with protrusive and contractive forces, respectively, the resulting anisotropy force fields (Fig. 2c) drive cell migration, and the integrin-ligand bonds provided by the basic active integrins are stretched. The local mechanical-chemical feedback loop could be initiated if the tensile force bypasses a threshold. Thus, more active integrins are produced from the pool of inactive integrins, which, in turn, accelerates cell migration by strengthening cell-substrate adhesion. Successively, the spatiotemporal regulation of both inactive and active integrins reaches a steady state (Fig. 2d). Here, active integrins present biased distributions due to the increased local ampli- fication from the mechanical-chemical feedback loop and limited diffusivity (Fig. 2d, bottom), while inactive integrins are distributed uniformly (Fig. 2d, top). By contrast, no biased distribution is found for bound integrins (Fig. 2e), suggesting that the accumulation of active integrins is not strong enough to introduce anisotropic cell-substrate adhesion in the absence of directional Rac activity. Time courses of cell velocity in this case (red line) and in two other cases (brown and blue lines) are also presented in Fig. 2f. In these typical cases, the cell undergoes the transition of an initial increase $(0-40 \mathrm{~s})$, a linear increase $(40-400 \mathrm{~s})$, and a stable plateau (400- $700 \mathrm{~s}$ ) along with the spatiotemporal regulation of PIs and active integrins. On the other hand, since the cell merely develops its motility by amplifying stochastic noise, these time courses of cell velocity naturally manifest substantial differences upon each simulation (Fig. 2f).

\subsection{Modeling chemotactic migration}

To further simulate the chemotactic behaviors of neutrophils, the substrate stiffness is set as described above, and a micropipette loaded with an fMLP solution is added close to the cell, a source point with a constant fMLP concentration [Eq. (2)]. The system runs for $350 \mathrm{~s}$, and the cellular responses and spatiotemporal evolutions of key signaling components are recorded. Five seconds after introducing the source point, a steady fMLP field with a moderate gradient (7.65-8 nM) is formed surrounding the cell (Fig. 3a). The uniform distribution of GPCRs along the membrane allows the cell to sense the spatial difference in the fMLP concentration. However, the profile of occupied GPCRs at any time point (i.e., $t=50 \mathrm{~s}$ in Fig. $3 \mathrm{~b}$ ) inevitably displays strong stochastic noise due to the reversible binding/unbinding events. The stochastic signal received is quickly amplified by cytoskeleton-independent signaling cascades that are modeled by the balanced-inactivation mechanism [Eq. (3)]. Consequently, the spatial distributions of membrane components, represented by $G_{\beta \gamma}$ (activator) and $G_{\alpha}$ (inhibitor), mark visible front and rear zones (Fig. S2a and b), which further transfer a clear spatial message to the downstream signaling layer of small Rho GTPase regulation by providing the binding sites for PAK1 and Lsc1, respectively (Fig. S2c and d).

The spatial profiles of the three members in the steady state are illustrated in Fig. 3c for the sake of clarity. These bipolar distributions of active Rho GTPase members sufficiently evoke the spatial effects of Rho GTPase-PI-mediated feedback loops to control cytoskeletal remodeling. Particularly, higher Rac activity at the cell front provides a higher activating rate of membrane-bound PI3K, whereas higher RhoA activity at the cell rear offers a higher activation rate of membrane-bound PTEN. As the $\mathrm{PIP}_{3}$ level is evaluated by active PI3K at the front and decreased by PTEN 
Time (s)

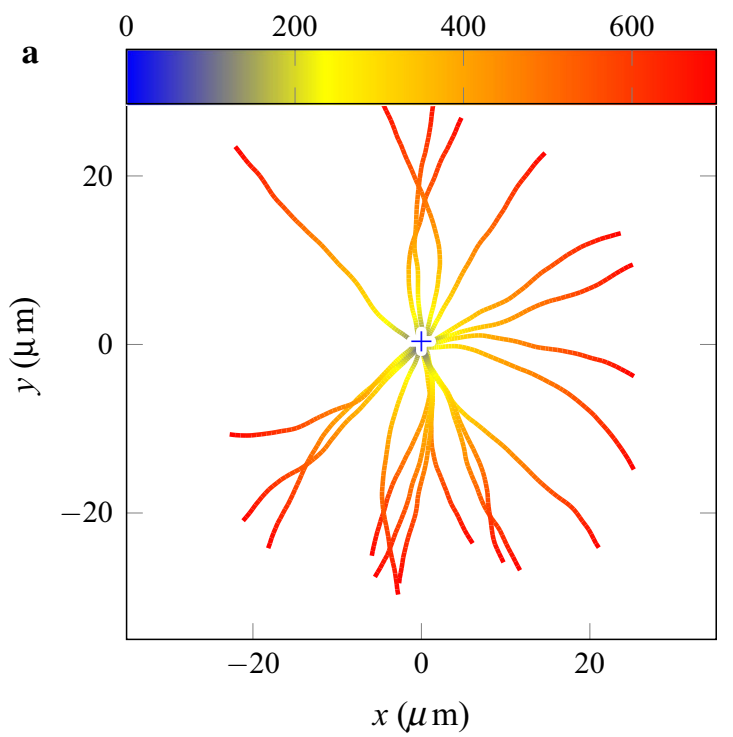

c
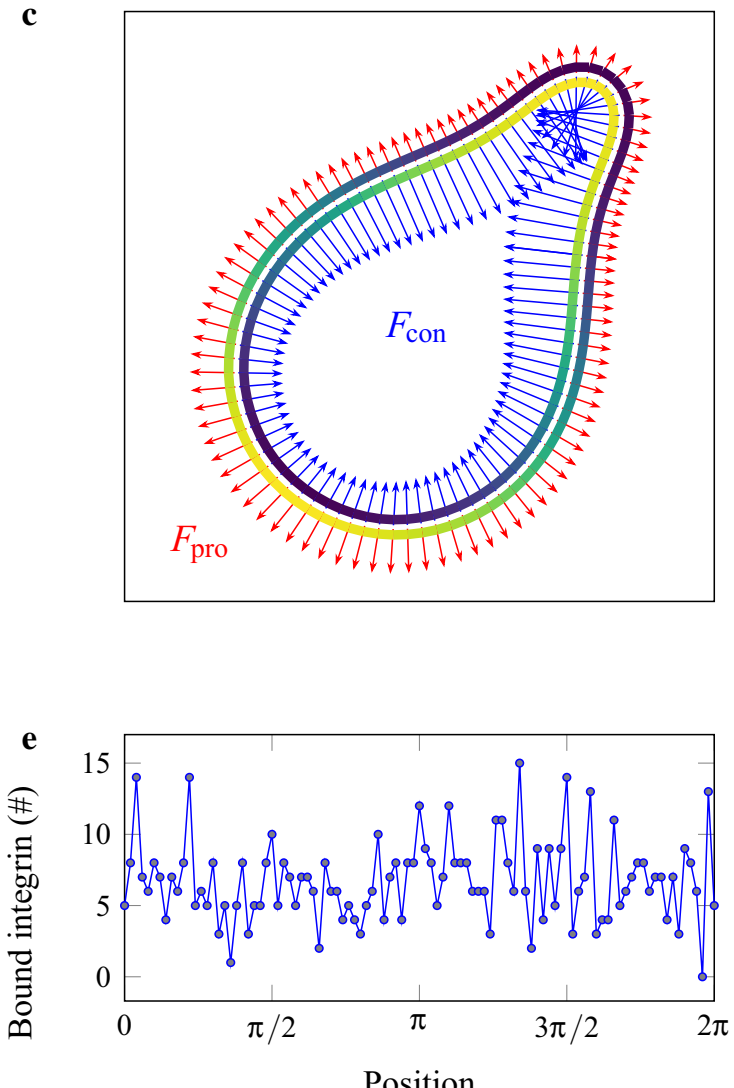

Fig. 2 Spontaneous neutrophil migration in the absence of a graded fMLP stimulus. a Cell centroid trajectories from 20 simulation runs for $700 \mathrm{~s}$ each. The cross denotes the starting point. b Spatiotemporal responses of $\mathrm{PIP}_{3}$ (upper) and $\mathrm{PIP}_{2}$ (lower) from a typical simulation. c Protrusive (outward) and contractive (inward) force fields calculated at $t=200 \mathrm{~s}$. Local protrusive $\left(\mathbf{F}_{\text {pro }}\right)$ and contractive forces $\left(\mathbf{F}_{\mathrm{con}}\right)$ are assumed to be proportional to the concentrations of $\mathrm{PIP}_{3}$ and $\mathrm{PIP}_{2}$, respectively (see Model assumptions in Supporting Information). d Spatiotemporal responses of inactive (upper) and active (lower) integrins. As the cell moves, the stretching of integrin-ligand bonds initiates

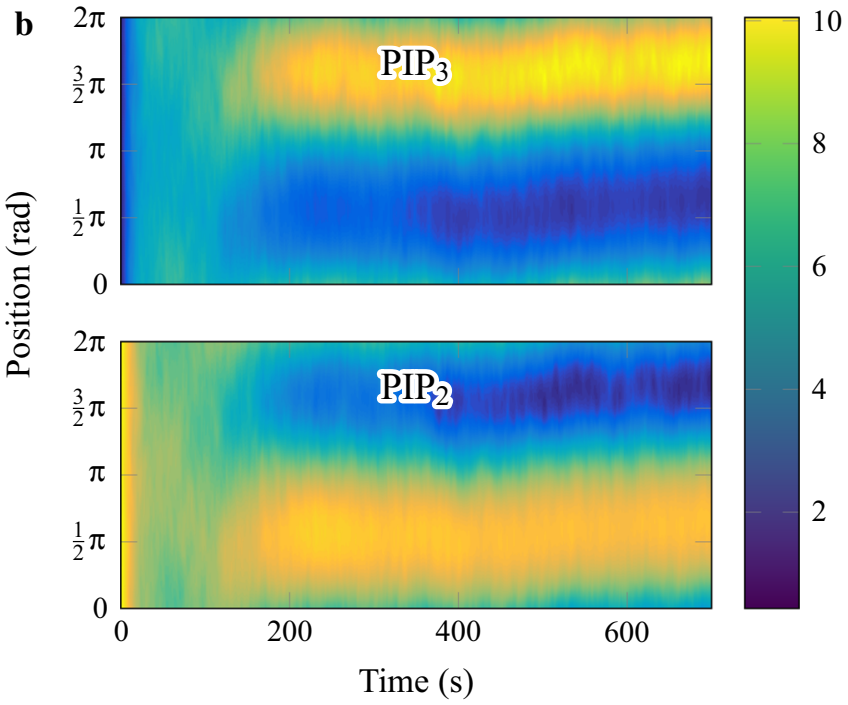

\section{0}

8

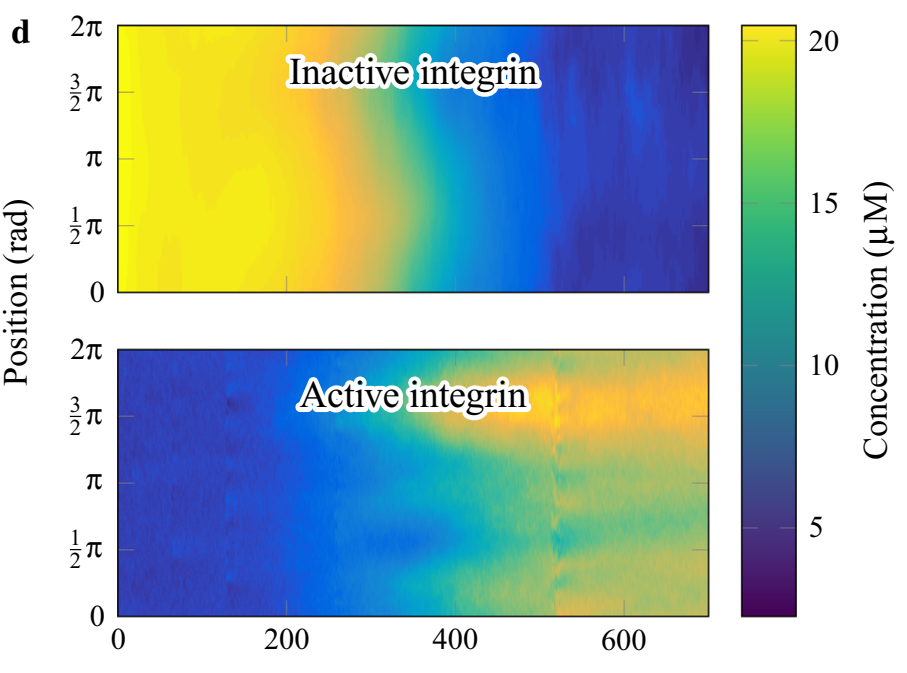

Time (s)

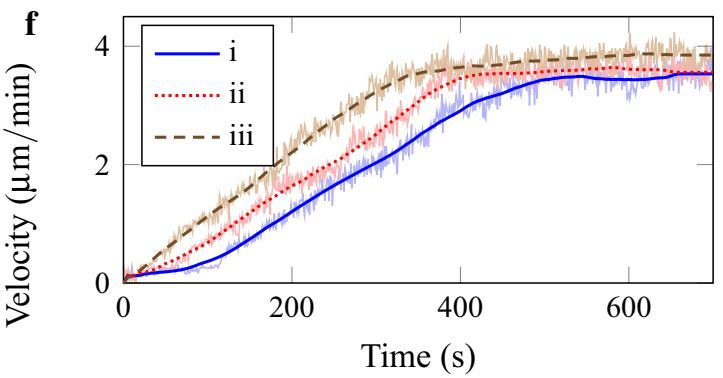

mechano-sensing pathways, thus transferring more active integrins than inactive integrins. e Profile of bound integrins along the cell periphery at the end of a typical simulation. Note that no polarized distribution is observable, suggesting that the extent of active integrin accumulation is not sufficient to mediate anisotropic adhesion. $\mathbf{f}$ Time courses of cell velocities for three randomly selected simulations presented in blue, red, and brown. In the absence of directional cues, the establishment of cell polarity is triggered by stochastic fluctuations such that the procedures for developing cell motility differ from each other 

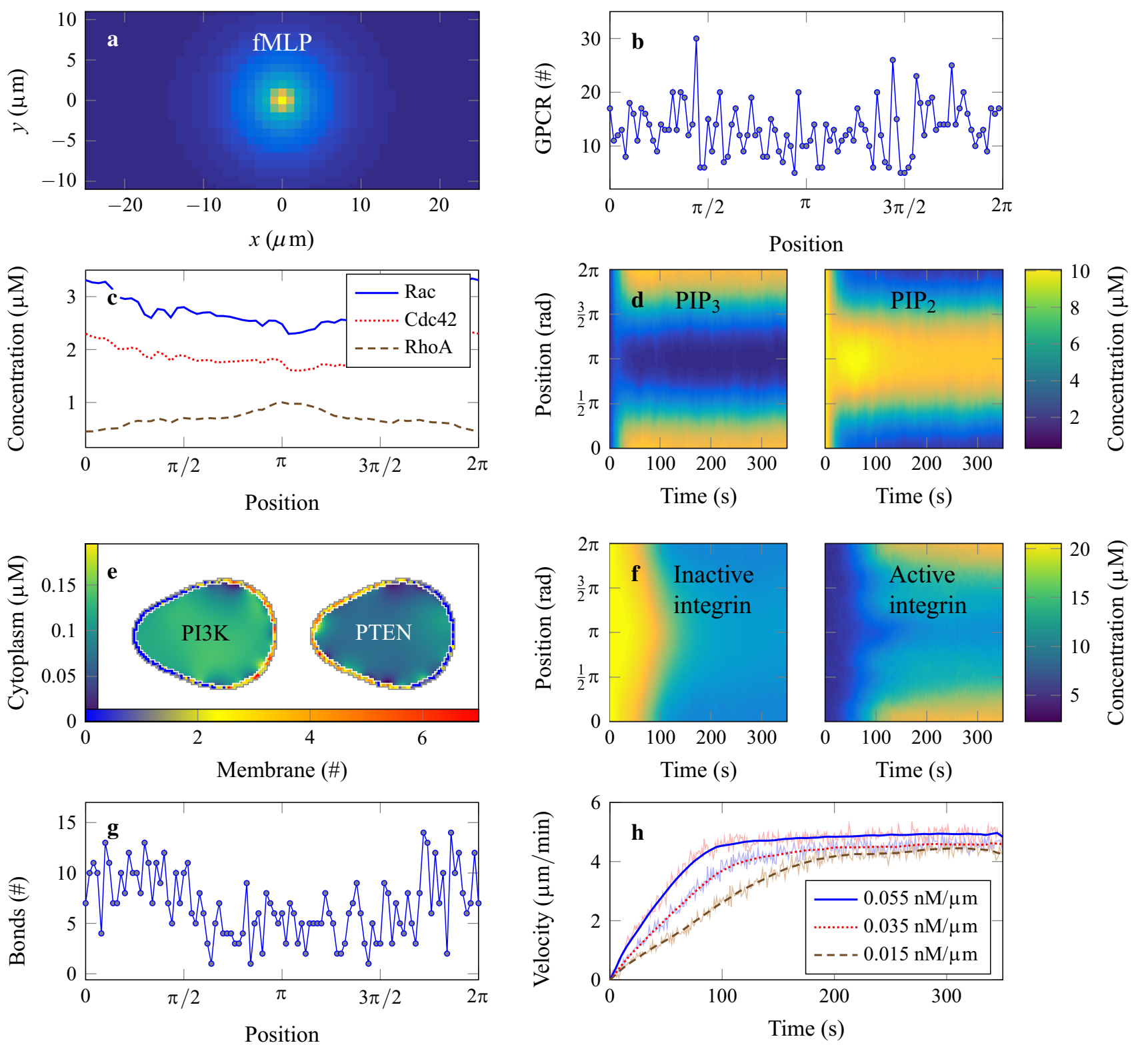

Fig. 3 Directional neutrophil migration upon application of a gradient fMLP stimulus. a Spatial distribution of the fMLP field in the steady state. The source point, mimicking a micropipette containing fMLP, is applied in a spatiotemporally varied manner. b GPCR occupancy profile along the periphery of a cell at $t=100 \mathrm{~s}$. The cell reads extracellular directional signals by evaluating local GPCR occupancy with a displayed strong noise. $\mathbf{c}$ Concentration profiles of active Rho GTPase members Rac (blue), Cdc42 (red), and RhoA (brown) in the steady state. Due to the antagonistic effects among the members and the spatial regulation effect of PAK1 and Lsc1, both active Cdc42 and Rac have higher concentrations at the cell front facing the fMLP source point, while active RhoA is centered on the opposite side. d Spatiotemporal responses of $\mathrm{PIP}_{3}$ (left) and $\mathrm{PIP}_{2}$ (right). Bipolar distribution patterns of Rho GTPase members evoke the spatial effects of Rho GTPase-PI feedback loops that drive bidirectional molecular transport, thus resulting in the all-or-none distributions of $\mathrm{PIP}_{3}$ and $\mathrm{PIP}_{2}$. e Spatial distribu-

tions of membrane-bound and cytosolic PI3K (left) and PTEN (right). Cytosolic PI3K and PTEN diffuse evenly throughout the inner region of the cell, while the distributions of their membrane-bound forms are in accordance with those of $\mathrm{PIP}_{3}$ and $\mathrm{PIP}_{2}$, respectively. $\mathbf{f}$ Spatiotemporal responses of inactive (left) and active integrins (right). The mechanicalchemical feedback loop initiated by stretching the integrin-ligand bond is spatially amplified upon activation of the Rac gradient (Block et al. 2016), and active integrins consequently become shapely localized at the cell front. $\mathrm{g}$ Bound integrin profile along the periphery of a cell at $t=200 \mathrm{~s}$. The strong accumulation of active integrins at the cell front leads to a polarized distribution of bound integrins, suggesting that the cell may establish firmer adhesion at the front. $\mathbf{h}$ Time courses of cell velocities for three simulations in which the initially applied fMLP gradient varies from 0.0055 (blue), to 0.0035 (red) to 0.0015 $\mathrm{nM} / \mu \mathrm{m}$ (brown). Increasing the fMLP gradient accelerates the process of achieving stable movement 
at the rear, a baseline intracellular $\mathrm{PIP}_{3}$ gradient is formed in accordance with that of extracellular fMLP. By providing the binding sites for cytosolic PI3K on the cell membrane, the gradually sharper $\mathrm{PIP}_{3}$ gradient drives the forward transport of PI3K in the cytosol. Correspondingly, since PIP 2 has been replaced by $\mathrm{PIP}_{3}$ at the cell front, membrane-bound PTEN tends to enter the cytosol. These PTEN molecules reach the rear via backward cytosolic diffusion, wherein they rebind with $\mathrm{PIP}_{2}$. This bidirectional molecular transport process ceases when cytosolic PI3K is significantly depleted. By then, all-or-nothing PI distributions are formed; membrane-bound PI3K and $\mathrm{PIP}_{3}$ accumulate at the front, while membrane-bound PTEN and PIP $_{2}$ accumulate in the rear (Fig. 3d and e). Accordingly, the cell generates active protrusive/contractive forces, becomes elliptical, and moves along the fMLP field gradient. On the other hand, the biased distribution of active Rac evokes the spatial effects of the mechanical-chemical feedback loop responsible for integrin activation. The local difference in Rac activity can be transferred into that of integrin activity. As a result, active integrins constantly accumulate at the front of the cell (Fig. $3 f$, right). This process ceases until the pool of inactive integrins becomes depleted, and a spatially biased distribution of bound integrins is formed (Fig. 3f and g), suggesting that the cell tends to establish firmer cell-substrate adhesion at the front. Time courses of cell velocity in this case (blue line) and in two other cases (brown and red lines) wherein the input fMLP gradient is either decreased or increased are also presented (Fig. 3h). The average migration rate of neutrophil is reported to be $0.059 \pm 0.005 \mu \mathrm{m} / \mathrm{s}$ (around $30 \mu \mathrm{m} / 700 \mathrm{~s}$ ) (Weckmann et al. 2017), which is comparable to the rate provided by our simulations and our verification experiments $(\sim 24.5 \mu \mathrm{m} / 700 \mathrm{~s})$ (Fig. S3, Video S1 and S2). Increasing or decreasing the input fMLP gradient tends to accelerate or decelerate the process, respectively.

\subsection{Impacts of substrate stiffness and integrin-ligand binding kinetics}

In addition to cytoskeletal remodeling, focal adhesion dynamics are another key factor that constrain neutrophil motility, and both processes typically occur in a coordinated manner. Despite the complexities of the aforementioned molecular mechanisms, the strength of focal adhesion is proportional to the local concentration of active integrins, which is controlled not only by intracellular biochemical factors, i.e., Rac activity (Block et al. 2016), but also by extracellular mechanical factors, i.e., substrate stiffness and integrin-ligand binding affinity itself (Peng et al. 2012).

In this section, cells expressing different types of integrins are set to undergo directional movement upon the application of substrates with varying stiffness. In each simulation, the same graded chemotactic stimuli are applied, and the displacement and shape of the cells are monitored at given time intervals. Cell velocity at the steady state is plotted against substrate stiffness and integrin-ligand binding kinetics (Fig. 4a). Generally, the estimated cell velocity is a biphasic function of substrate stiffness, which can be interpreted as follows: Cell motility results from balance among cellular adhesion strength, contractive force, and protrusive force, which are associated with the force built upon integrinligand bonds. This molecular-level force should either exceed a threshold that is required for successful integrin activation when more integrins become activated or reduce the dissociation rate of integrin-ligand bonds, based on Bell's model (Bell 1978). Upon application of a certain substrate stiffness (i.e., $E_{\text {sub }}>10 \mathrm{kPa}$ ), the buildup of the force is efficient [as explained by a serial two-spring model (Schwarz et al. 2006)], yielding a high probability of a bond beyond the threshold (Fig. S4a). Since a higher level of integrin activation corresponds to firmer cell-substrate adhesion, the magnitude of active forces is saturated. Further increasing the substrate stiffness evaluates the drag force and eventually results in diminished cell motility. In contrast, since the bond force buildup is not sufficient on soft substrates (i.e., $E_{\text {sub }}<2 \mathrm{kPa}$ ), the relatively weak cell-substrate adhesion restricts the magnitude of the active force, and the cell velocity is thus decreased. Unsurprisingly, cells generate maximal migration velocity only at intermediate substrate stiffness.

Additionally, the dependence of cell velocity on substrate stiffness differs based on specific integrin-ligand binding kinetics, suggesting that the expression of integrin variants acts as an adaptation mechanism to varied substrate stiffness. sMac-1 (red line), where the highest velocity occurs at $\sim 3 \mathrm{kPa}$, serves as an example comparison. Here, LFA1 yields higher dissociation kinetics with ICAM-1, and the estimated highest velocity shifts left upon the addition of a softer substrate (at $\sim 2 \mathrm{kPa}$, brown line) because the mechanical-chemical feedback loop is efficiently evoked upon the inclusion of softer substrates for integrins that possess lower dissociation kinetics. By contrast, Mac-1 processes lower dissociation kinetics with ICAM-1, and the highest velocity shifts right upon the addition of a stiffer substrate (at $\sim 5 \mathrm{kPa}$, blue line), as expected. Since the dissociation kinetics of specific integrins govern the level of active integrins at distinct substrate stiffness values, the association kinetics further determine the amount of bound integrins, and thus, the cell velocity.

The cell morphologies and active integrin distributions at the steady state are exemplified in Fig. 4b. For three pairs of two integrins (Mac-1, sMac-1, and LFA-1) with distinct binding kinetics, increasing substrate stiffness enhances integrin activation; the global level of active integrins is low $(\sim 3 \mu \mathrm{M})$ on a soft substrate $(1 \mathrm{kPa})$ and reaches saturation on intermediate $(5 \mathrm{kPa})$ and stiff substrates $(20 \mathrm{kPa})$. The shape of 


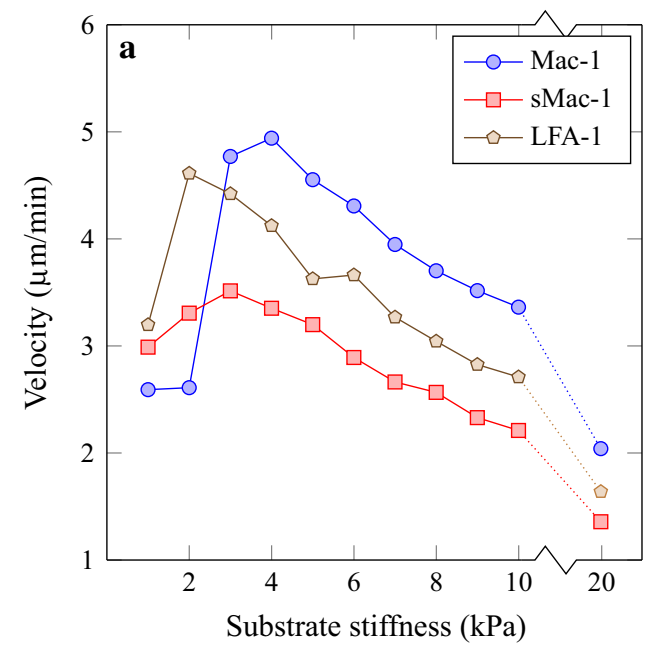

Fig. 4 Impacts of substrate stiffness and integrin-ligand binding kinetics on neutrophil migration. The substrate stiffness varies from 1 to 20 $\mathrm{kPa}$. Three sets of binding kinetics $\left(k_{\mathrm{off}}^{0}(\mathrm{~s}), A_{\mathrm{C}} k_{\mathrm{on}}\left(10^{-6} \mu \mathrm{m}^{4} \mathrm{~s}^{-1}\right)\right)$, including Mac-1-sICAM-1 (1.12, 0.90) (blue in a), sMac-1-ICAM-1 $(0.43,0.39)$ (red in a), and LFA-1-ICAM-1 $(0.5,0.5)$ (brown in a), are given in the simulations. a Average migration velocity as a function of substrate stiffness and binding kinetics. In general, the stiffness dependence of cell velocity is biphasic. Altering the binding kinet-

the cell is mainly regulated by the substrate stiffness. Cell asymmetry is less evident on soft and stiff substrates due to the lack of adhesion or the formation of over-firm adhesion. The cell develops asymmetric morphology with a polarized front and rear on only an intermediate substrate. The spatiotemporal deformation patterns of a model cell expressing sMac-1 during its migration process on a $5 \mathrm{kPa}$ substrate are presented in Fig. S4b and c, indicating how the cell develops its contractive rear.

\subsection{Turnability of cell motility}

This model can be further extended to elucidate cell behaviors in response to reversal stimuli with varying steepness values. The simulations are started from the non-polarized cell state. The same initial stimulus is applied at $t=0 \mathrm{~s}$, and a specific reversal stimulus is applied at $t=350 \mathrm{~s}$ for an additional 550 $\mathrm{s}$. The cell centroid trajectories (colored lines) and snapshots of cell shape (colored by $\mathrm{PIP}_{3}$ concentration) are presented in Fig. 5. In response to a steep reversal gradient (i.e., 0.095 $\mathrm{nM} / \mu \mathrm{m}$ ), the cell approaches the new stimulus by reversing its original motility in a nearly straight trajectory (Fig. 5a and Video S3). Interestingly, since the cell first loses its original polarity and then repolarizes correctly in accordance with the new stimulus direction, a remarkable time lag exists (e.g., from 350 to $405 \mathrm{~s}$ ) during which the cell returns to a nonpolarized, round state, and its net motility is nearly abolished. At a moderate reversal gradient (i.e., $0.055 \mathrm{nM} / \mu \mathrm{m}$ ), the cell

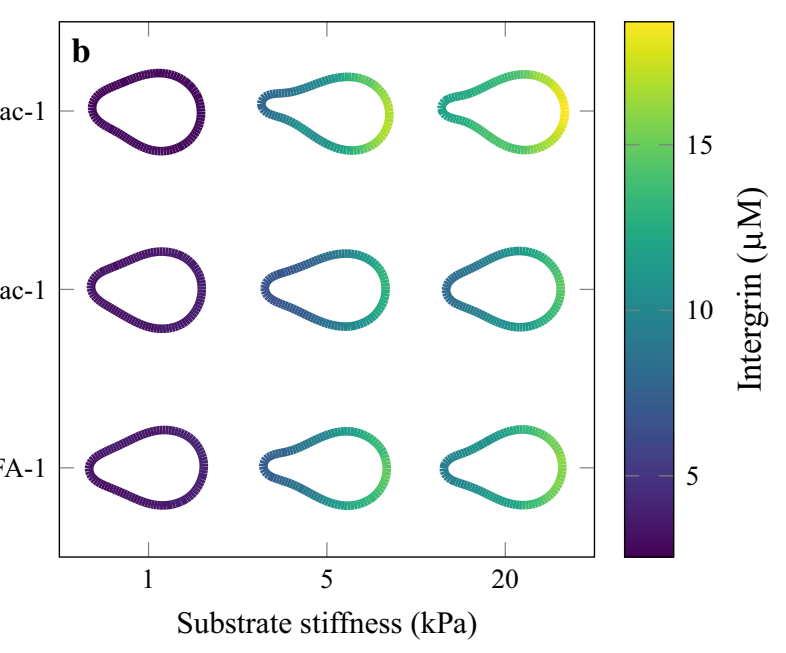

ics shifts the location of the peak velocity value, suggesting that the expression of distinct integrins serves as an adaption strategy upon the addition of different substrates. b Morphologies and integrin responses at varying substrate stiffness values and reaction kinetics. As the stiffness increases, the activation of integrins becomes saturated due to the increased efficiency of triggering mechanical-chemical feedback. The cell is more asymmetric on intermediate substrates $(5 \mathrm{kPa})$ and less so on soft $(1 \mathrm{kPa})$ and stiff $(20 \mathrm{kPa})$ substrates similarly maintains its original movement direction during the time lag period, but the polarity of the cell yields a slow rotation rather than a direct reversal (Fig. 5 b and Video S4). Consequently, the cell makes a U-turn in response to the change in the gradient direction. When a shallow reversal gradient is applied (i.e., $0.035 \mathrm{nM} / \mu \mathrm{m}$ ), the cell maintains its migration along its original direction (Fig. $5 \mathrm{c}$ and Video S5).

To elucidate how cell motility can be fundamentally tuned by the steepness of the reversal stimulus, the spatiotemporal patterns of $\mathrm{PIP}_{3}$ and the time courses of cytosolic PI3K/PTEN amounts corresponding to various gradients are presented in Fig. S5. When a cell receives stimuli from the opposite direction, Rho GTPase members effectively redistribute their activities; the activated Rac and $\mathrm{Cdc} 42$ are detected at the incipient front and reduced at the incipient rear, while RhoA acts oppositely. Since Rho GTPase members (in their active forms) yield faster redistributing dynamics than PIs and steeper reversal stimuli result in sharper Rho GTPase gradients (data not shown), the location where the highest Rac $\rightarrow$ $\mathrm{PI} 3 \mathrm{~K} \leftrightharpoons \mathrm{PIP}_{3}$ positive-feedback loop strength occurs could be varied by specific reversal stimulus gradients. Accordingly, different spatiotemporal patterns of $\mathrm{PIP}_{3}$ are produced (Fig. S5a-c). By monitoring the time course of cytosolic PI3K amounts (Fig. S5d), a quantitative understanding of cell turnability can be achieved. Once the stimulus is reversed, the evaluation of RhoA activity at the incipient front reduces local $\mathrm{PIP}_{3}$ via active PTEN, which induces the dissociation of 

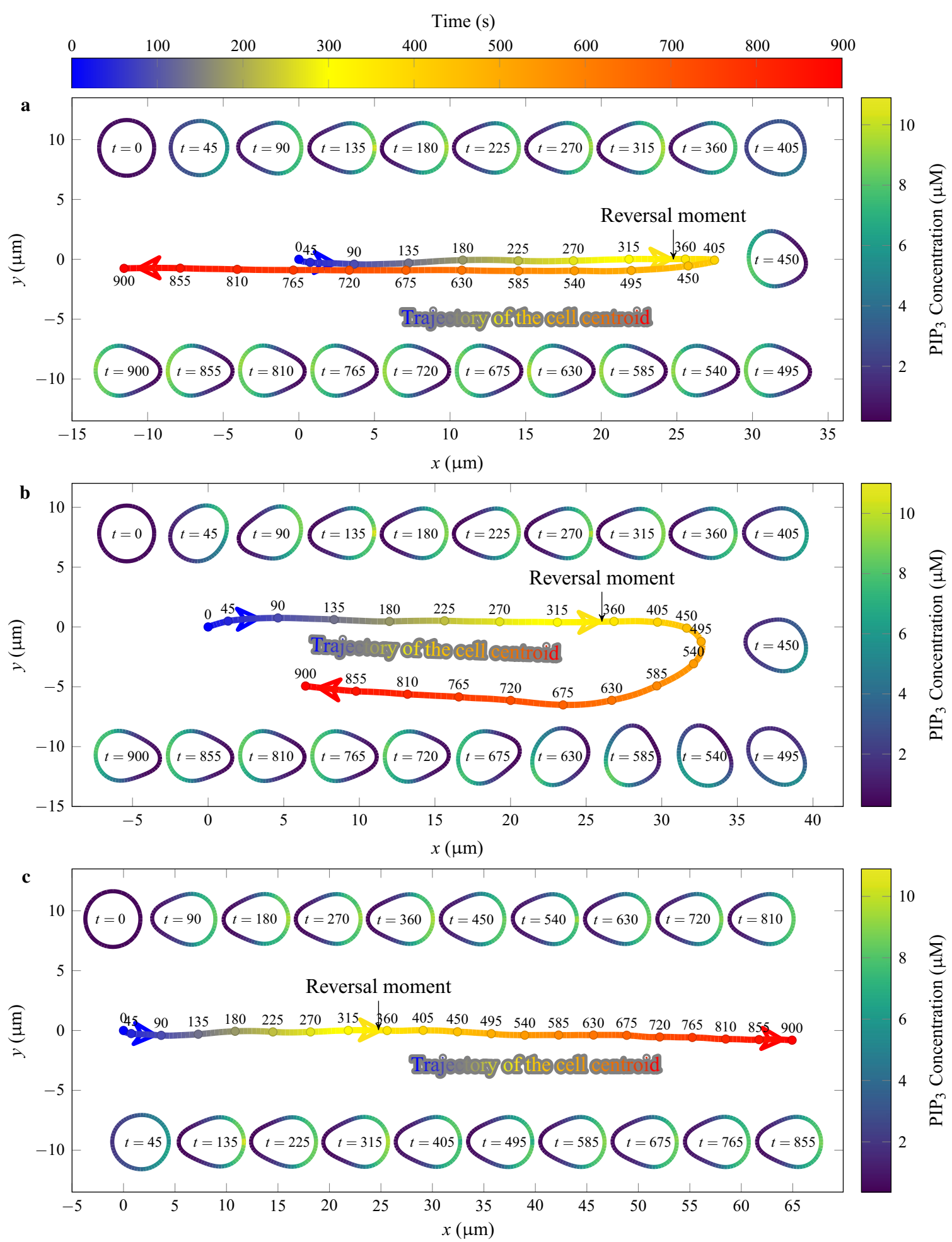

Fig. 5 Influence of reversal gradient steepness on neutrophil turnability. The cell originally set in a fMLP gradient and moving to the right receives the reversal fMLP stimulus at $t=350 \mathrm{~s}$ at different reversal gradient steepness values. Time-lapsed snapshots of the cell trajectories and shapes are illustrated. The colored bar on the right denotes $\mathrm{PIP}_{3}$ concentrations. a Responses of a cell to a steep reversal stimulus $(0.095$ $\mathrm{nM} / \mu \mathrm{m}$ ); the cell reorients itself by first losing its original polarity and then reestablishing its $\mathrm{PIP}_{3}$ polarity toward the new stimulus. Note that a time lag period exists (from 350 to 405 s) during which $\mathrm{PIP}_{3}$ accumulation nearly disappears (also see Video S3). b Responses of a cell to a moderate reversal signal $(0.055 \mathrm{nM} / \mu \mathrm{m})$, where $\mathrm{PIP}_{3}$ redistribution is achieved in a slow rotation manner such that the cell prefers to make a U-turn to the new stimulus (also see Video S4). c Response of a cell to a shallow reversal signal $(0.035 \mathrm{nM} / \mu \mathrm{m})$, where $\mathrm{PIP}_{3}$ accumulation is frozen such that the cell becomes stuck in its original direction (also see Video S5) 
membrane-bound PI3K and thus increases the cytosolic PI3K profiles (Fig. S5d). Specifically, in response to steeper reversal stimuli, the strong rejection effect of membrane-bound PI3K, elevated from the increased RhoA activity at the original cell front, results in the fast increase in cytosolic PI3K amounts (blue line, 350-410 s). Accordingly, the original rear efficiently accesses cytosolic PI3K and forms the newly born pseudopod corresponding to the decrease in the PI3K profile (blue line, 410-470 s). When the reversal stimulus gradient decreases, the increase in cytosolic PI3K becomes slow (red line, 350-490 s) or completely ceases (brown line). The previously formed pseudopod still runs such that the cell displays a "U-turn" or "lock-on" behavior.

\subsection{Responses of mutated cells}

This model is also applied to test a typical biological scenario for the responses of cell mutants with distinct PTEN expressions. Using the same time-dependent initial and reversal fMLP stimuli (cf. Fig. 5a), the corresponding cell trajectories and the cell shapes are presented at a series of successive time points (Fig. 6). PTEN-overexpressed cells (i.e., $[\mathrm{PTEN}]=0.14 \mu \mathrm{M})$ require more time to accumulate $\mathrm{PIP}_{3}$ and become fully polarized (Fig. 6a) because the inhibiting effect of cytoskeletal remodeling in mutated cells is stronger than that in wild-type cells. In addition, these mutants have lower migration velocities, exhibiting reductions of $\sim 70 \%$ compared with those of wild-type cells. By contrast, cells become easily excited when PTEN is deficient (i.e., [PTEN] $=0.035 \mu \mathrm{M})$. The shortage of the inhibitor allows the cell to generate a pseudopod along its entire periphery. Here, the mutated cell is pushed at both the front and the rear, restricting its migration velocity. Meanwhile, the cell has a defective contractive rear (Fig. 6b).

Once the reversal stimulus is received, the mutant cells respond differently. For example, a PTEN-overexpressed cell tends to return to a non-polarized state (Fig. 6a, 350900 s). Although a high PTEN expression level results in a remarkable inhibiting effect of membrane-bound PI3K at the original cell front, the relative ratio of [PTEN] to [PI3K] in the cytosol is sufficiently high enough that the cell fails to be repolarized. On the other hand, relatively excessive PI3K expression promotes the cell to establish its new front directly opposite from where PTEN is deficient (Fig. 6b). Collectively, the above simulations imply that maintaining proper levels of core signaling molecules in the cell is substantially important. Since the role of PI3K in mediating PI signaling is opposite to that of PTEN, cells overexpressing PI3K may exhibit behaviors similar to those of PTEN-deficient cells and vice versa.

\section{Discussion}

To elucidate the migration behaviors of neutrophils in different physiological environments, we herein proposed a mechanochemical model with an intermediate level of molecular details. The model is multi-modularized, containing a four-layered signaling module, a focal adhesion regulation module for mechano-sensing, and a motility module. By exploring the integrative model, we gained the following insights that help to understand the mechanisms of neutrophil chemotaxis: First, the four-layered signaling structure with the strongest feedback at the bottom layer can properly capture the spatiotemporal regulation of intracellular molecules during neutrophil migration (Figs. 2 and 3). Second, the expression of integrin variants is considered an adaptation strategy for efficient neutrophil migration upon the application of substrates of varying stiffness (Figs. 4 and S4). Third, rather than treating cytosolic effectors as homogeneous species, as assumed in earlier modeling studies (Postma and Haastert 2001; Onsum and Rao 2007), this model demonstrates that proper cytosolic diffusion generates dual effects, effective molecular transport and moderate molecular trapping, both of which are required for appropriate cell reorientation (Fig. S6). Finally, the generation of different neutrophil turning behaviors (i.e., reversal, Uturn, and lock-on) is attributed to the synergistic effects of instantaneous intracellular molecular transport and the original cellular movement (Figs. 5 and 6). To further explain the above insights in more detail, three key issues, (i) core principals for constructing our model, (ii) comparisons to experimental data and early modeling studies, and (iii) limitations of our model, are discussed below.

\subsection{Core principles for constructing our model}

One of the most theoretical difficulties in simulating cell motility is how to adequately describe the complicated signaling cascades responsible for cytoskeletal remodeling. Generally, these cascades are organized into a network that describes the relationship among different molecules. Cells treated with actin polymerization inhibitors become rounded and lose polarity, yet they continue to sense the direction of chemotactic gradients (Van Haastert and Devreotes 2004). By contrast, the exogenous activation (Lin et al. 2012) or delivery of signaling molecules (Weiner et al. 2002) while bypassing upstream cascades may induce chemotactic behaviors without biochemical stimuli. These experimental data imply that the signaling cascades responsible for cytoskeletal remodeling could be multilayered. From this viewpoint, the signaling process can be deciphered as follows: First, a neutrophil utilizes the GPCR occupancy to depict the local concentration of the surrounding biochemical field (signal reception, Fig. 1a-i). Second, G protein dis- 
Time (s)
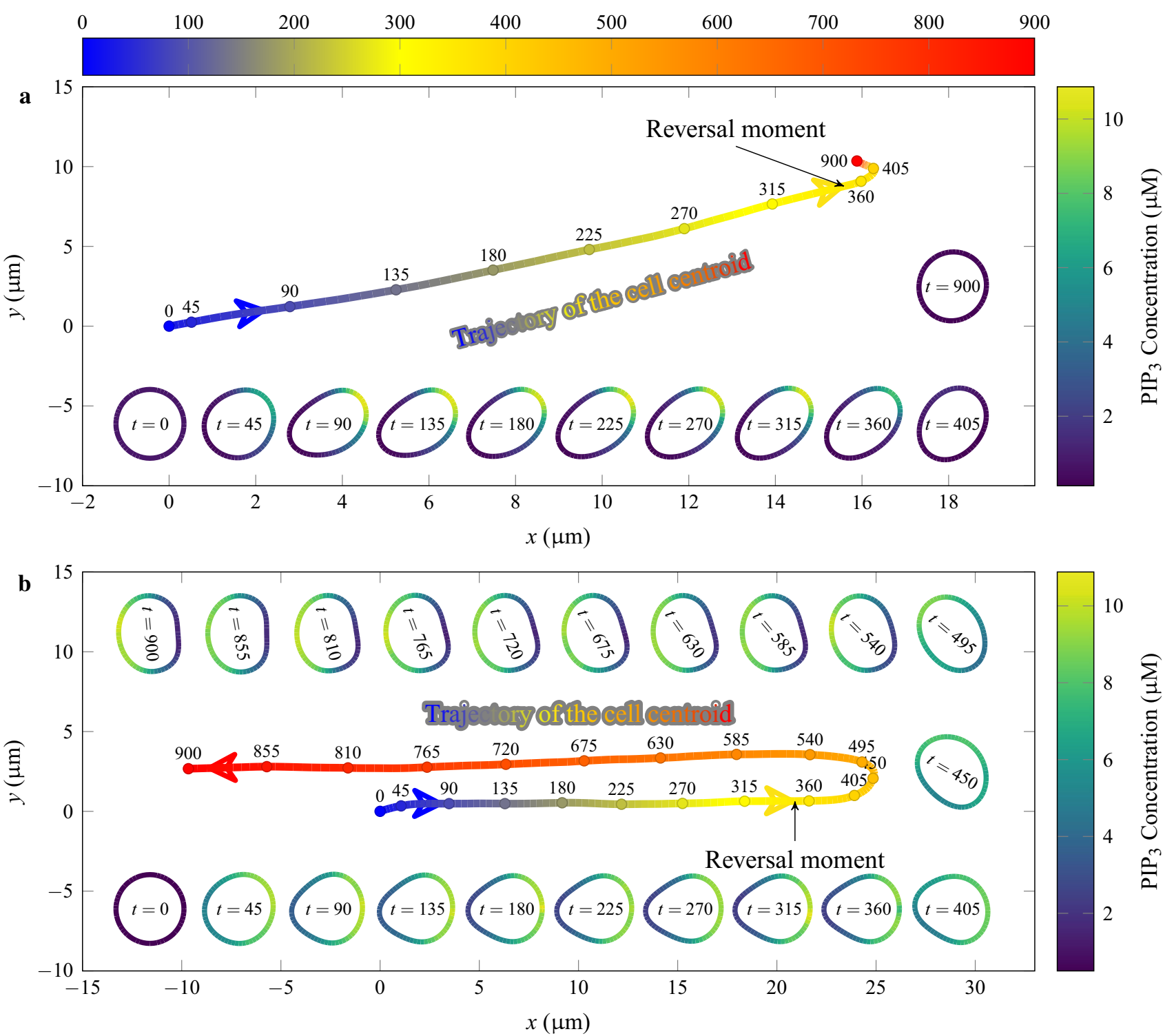

Fig. 6 Responses of a PTEN-mutated cell to the same initial and reversal stimuli. Initial and reversal stimuli gradients are set to 0.035 and $0.095 \mathrm{nM} / \mu \mathrm{m}$, respectively. Cellular responses vary dramatically upon the alteration of PTEN expression. a Cell centroid trajectory and $\mathrm{PIP}_{3}$ response to a $200 \%$ increase in the basal PTEN basal. The cell accu- mulates a small amount of $\mathrm{PIP}_{3}$, corresponding to the development of a small pseudopod, and fails to repolarize in response to the secondary stimulus. b Trajectory of a cell centroid and $\mathrm{PIP}_{3}$ response to a 50\% decrease in the basal PTEN level. The cell establishes a wide range of $\mathrm{PIP}_{3}$ accumulations and fails to develop a contractive rear sociates into $G_{\alpha}$ and $G_{\beta \gamma}$ subunits, which are responsible for regulating fast activation and slow inhibition responses, respectively (Levine et al. 2006). Meanwhile, the diffusion of signaling molecules on the membrane effectively eliminates the noise from the received signal (initial signal processing, Fig. 1a-ii). Third, GEF localization along with the antagonistic effects of Rho GTPase members promotes Rho GTPase bipolar distribution patterns (Rho GTPase regulation, Fig. 1aiii) (Raftopoulou and Hall 2004). Fourth, short-range positive and long-range negative feedback loops are generated from the depletion of effector molecules in the cytosol and the interconversion of substrate molecules (i.e., $\mathrm{PIP}_{3}$ and $\mathrm{PIP}_{2}$ ) at the membrane (Kölsch et al. 2008), resulting in molecular transport in one direction enhancing the transport in the opposite direction (bidirectional molecular transport, Fig. 1a-iv). Based on our previous studies (Feng and Zhu 2014; Feng et al. 2018) as well as those from other laboratories (Marée et al. 2012; Otsuji et al. 2007), the mechanisms that enable the spatial separation of Rho GTPase members and PIs (i.e., $\mathrm{PIP}_{3}$ and $\mathrm{PIP}_{2}$ ) are different. The former is caused by 
mutually exclusive effects between Rho GTPase members, as modeled by double-negative feedback loops. The latter is induced by the spatial effects of Rho GTPase-PI-mediated feedback loops. Physically, it also relies on the translocating features of the effectors (i.e., PI3K and PTEN) along with fast molecular diffusivity in the cytosol. Our model results verified that once the clear all-or-nothing PI distribution pattern is established, the actin network is pushed at the front and pulled at the rear such that the cell achieves the most efficient movement upon the guidance of shallow, graded biochemical stimuli. In the absence of directional stimuli, however, the generation and growth of such $\mathrm{PIP}_{3} / \mathrm{PIP}_{2}$ patches rely on only the amplification of stochastic noise, and achieving significant cell motility thus requires more time.

This work also proposes a novel numerical method, LBP, which is helpful for solving MBPs of inherently deformable, non-stationary domains with redistributed biochemical signaling components. In the past two decades, several computational frameworks for MBPs have been proposed. Although these frameworks differ in their computational methodologies, their procedures are similar (Holmes and EdelsteinKeshet 2012). In principle, two separate aspects are required. First, a method of choice, such as the level-set (LS) method (Neilson et al. 2011; Shi et al. 2013), phase-field (PF) method (Camley et al. 2013), or cellular Pott (CP) method (Marée et al. 2012, 2006), is implanted to track the moving boundary. Second, any conventional numerical method, such as the finite difference (FD) method, finite element (FE) method, or finite volume (FV) method, is invoked to solve the interior reaction-diffusion problem. Comparably, in the current LBP method, the particle method is applied to specify the moving boundary conditions via coupled, nonlinear reaction-diffusion equations, wherein the partial differential equations in the irregular domains are solved by the LBM. In a sense, the greatest advantage of the LBP method derives from implementation of the LB method (Ayodele et al. 2011). On the one hand, since the computational domain mesh is achieved using small, regular lattices, the specifications of spatiotemporally changed domains can be reached by means of an elementary Boolean operation. These features provide a simpler description of complicated geometry compared with those of conventional numerical methods. On the other hand, arithmetic operations in conventional computing are performed in only the collision process (see Supporting Information), which is a localized operation, such that the LB method-based MBP framework is substantially efficient and suitable for parallel processing.

\subsection{Comparison to experimental data and early modeling works}

Our model can quantitatively interpret a group of distinct but interrelated experimental neutrophil chemotaxis obser- vations. The first set of our cell chemotaxis simulations (Figs. 2 and 3) aims to compare the integrated model against the following basic aspects of experimental observations. The outputs of neutrophil migration experiments come from both cellular and molecular levels. At the cellular level, the neutrophil undergoes directional migration upon the application of graded stimuli or spontaneous migration upon the application of uniform stimuli (Nelson et al. 1975). At the molecular level, the spatial patterns of signaling molecules at different time points are presented (Wong et al. 2006; Gardiner et al. 2002). Although cellular observations have been captured by early modeling works, most rely on a single signaling mechanism achieved by abstract signaling components (Shi et al. 2013) or simple GTPase models (Wolgemuth et al. 2011; Vanderlei et al. 2011). Here, by adapting a four-layered approach to the complicated signaling cascades, we propose that when perceiving external stimuli in real time, isolation of the directional signal from strong noise, having two poles defined for actin self-organization, and effective bidirectional molecular transport are prerequisites for cytoskeletal remodeling in neutrophil chemotaxis. To this end, our model links experimental observations at the two levels. Models belonging to such multilayered categories do exist (Marée et al. 2012; Dawes and Edelstein-Keshet 2007), but they have differing dominant mechanisms for the different layers derived from distinct model cells.

The second set of simulations (Fig. 4) aims to compare with experimental data showing that the chemotaxis behavior of neutrophils is substrate dependent. Recent measurements using neutrophils (Stroka and Aranda-Espinoza 2009) as well as other eukaryotic motion cells, i.e., fibroblasts (Pelham and Wang 1997), smooth muscle cells (Peyton and Putnam 2005), and endothelial cells (Yeung et al. 2005), have demonstrated that the optimal cellular migration efficiency is achieved with the substrate of intermediate stiffness (5-10 kPa) (Janson and Putnam 2015). With stiff substrates ( $>20$ $\mathrm{kPa}$ ), the cell develops mature focal adhesions at both the front and the rear, which is too firm to break and therefore cannot effectively move. Conversely, with soft substrates $(<1 \mathrm{kPa})$, the cell forms a round morphological shape and presents unstable adhesions, which are unable to support the appreciable motility. Theoretically, our model is not the first to address this issue, as experimentally observed biphasic dependences of cell migration speeds on substrate stiffness have been previously explained merely by the force balance (Dokukina and Gracheva 2010; Gracheva and Othmer 2004). By contrast, our model and the related simulations are more biologically relevant, as they link cell migration speed and receptor-ligand binding kinetics. Unlike chemoattractant stimuli, alteration of the mechanical environment is fast within a short spatial range, and biological tissue stiffness reflects significant changes over large temporal and spatial scales in accordance with healthy or abnormal tissue (Janmey 
and Miller 2011). Correspondingly, as a neutrophil adapts to chemoattractant stimuli via spatiotemporal regulation with quick activation and slow inhibition, it also adapts to substrate stiffness via expressing various types of integrins.

The third set of simulations (Fig. 5) focuses on unraveling the specific features of cell turning. The turning behaviors of neutrophils in response to a secondary reversal stimulus have been investigated since 1981 (Gerisch and Keller 1981). Accordingly, two distinct turning forms are defined with similar timescales of 1-2 min, (i) U-turn (neutrophil follows the existing lamellipodium at the leading edge to make a stepwise movement toward the source) and (ii) reversal, (cell loses its previous lamellipodium, after which a new front is induced at the opposite end and points to the new attractant source). Monitoring the time courses of F-actin and myosin II fluorescence intensities detected a significant time delay (approximately $30 \mathrm{~s}$ ) in F-actin redistribution after reversing external stimuli (Dalous et al. 2008), providing signal processing evidence at the molecular level and the "sequence of reversal" at the cellular level. On the other hand, theoretically interpreting cellular turning behaviors is still diverse and not fully understood. For example, in the divergent pathway model (Postma and Haastert 2001) specific to neutrophils, the U-turn feature is explained by only the delayed response of intracellular signaling molecules, while the effect of the strength of the secondary stimulus is not considered. In a cellular Potts model (Marée et al. 2012) of steady keratinocyte motility, cell shape changes may feed back to intracellular signaling dynamics regulation, resulting in a faster U-turn rate. Compared with these early modeling works, our model replicates both "U-turn" and "reversal" turning features and reports the latter with the correct sequence. Such improvements are achieved by two factors. First, we successfully captured the intracellular signaling responses on a proper spatiotemporal scale (i.e., the PIs achieved the all-or-none distribution pattern in $2 \mathrm{~min}$ ) via the definition of signaling layers specific to neutrophils. Second, such specific chemotactic features are attributed to the molecular trapping effect induced by cytosolic diffusion and the stochastic translocation of effector molecules (Fig. S6). Overall, by combining with the motility module, the actual cell fate in chemotaxis is governed by competition between the mechanical strengths of retaining the existing lamellipodium and forming a new lamellipodium. Adopting the competitive strategy enables a neutrophil to choose its target most efficiently.

The final set of simulations (Fig. 6) aims to predict how a mutated cell migrates along the chemotactic gradient. Motivated by the prioritized role of PTEN in neutrophil migration, PTEN is selectively depleted in neutrophils with a loxPCre system wherein Cre recombinase is expressed under the control of the granulocyte-elastase promoter (Ela2CrePtenfl) (Heit et al. 2008). PTEN-deficient neutrophils produce two or more lamellipodia and an unidentifiable uropod. Correspondingly, Ela2CrePtenfl mice also show less cell recruitment during infection, which is manifested as a lack of motility in PTEN-deficient neutrophils. On the other hand, the behaviors of cells overexpressing PTEN are equivalent to those with reduced PI3K since the roles PI3K and PTEN are reciprocal. Blocking PI3K signaling using a wortmannin inhibitor shows that the cell exhibits a poorly developed lamellipodium, crawls much more slowly, and fails to persist in forward movement, which unlikely enables its migration all the way to the stimulus source point (Wang et al. 2002). These observations are consistent with our model predication in Fig. $6 \mathrm{~b}$ and a, respectively.

\subsection{Limitations of our model}

While our predictions are, in principle, consistent with those of experimental studies, and the proposed LBP method is suitable for our numerical calculations in MBPs, several issues remain. First, at least two aspects of the four-layered signaling module could be improved, one of which is in the initial signaling processing layer. Although introduction of the balanced-inactivation mechanism provides our model great simplicity, such integral treatment relying on abstract components could also be combined with other mechanisms, such as the local modulation of chemoattractant (Mackenzie et al. 2016) or a phase separation mechanism (Gamba et al. 2005). The other improvement involves the small Rho GTPase regulation layer, as only one Rho GTPase cross-talk scheme was selected from many other candidates for simplicity (Holmes et al. 2012; Jilkine et al. 2007), and this selection needs to be extended to other related signaling pathways (i.e., FilGAP) (Houk et al. 2012).

Second, while the focal adhesion regulation module is minimized to contain the two essential aspects of integrin regulation, i.e., the inside-out and outside signaling pathways (Yap and Kamm 2005), more detailed mechanosensing mechanisms are required to achieve a broad range of neutrophil motility. For example, numerical simulation has shown that membrane tension could regulate the growth of nascent focal adhesion (He and Ji 2016). In an integrin cluster model, the force-dependent integrin clustering is considered by local chemical potential reduction (Kong et al. 2010). From a theoretical modeling viewpoint, the local activation of an existing integrin has the same effect as integrin cluster. However, this microscopic model may provide a physical interpretation of the threshold force $\left(F_{\mathrm{r}}\right)$ adopted here. Besides, mechanical factors are well known to also affect the regulation of Rho GTPases, especially RhoA (Schaefer and Hordijk 2015). Myosin II-dependent contraction is activated by RhoA and Rhodependent kinase (ROCK) signaling, while the increased contraction consequently increases Rho/ROCK signaling 
(Rottner and Stradal 2011). Considering that a neutrophil moves relatively fast on soft substrates and no correlation between traction force and focal adhesion size is observed for mature adhesion (Stricker et al. 2011), the effect of a force-mediated RhoA signaling feedback loop is not a limiting factor for neutrophil motility in free space. However, such an effect may increase or even become dominant when neutrophils are required to enter a confined space [e.g., via a hepatic sinusoid or the ECM network (Cao et al. 2016)] whereupon the cell needs to enhance its contractility to override the resistance raised from a stiffer nucleus.

Additionally, the cytoskeletal remodeling module is quite simplified in our model. The shortage of earlier models in combination with previous observations in signaling cascade disruption experiments led to our proposal of a mechanochemical model of neutrophil chemotaxis based on signaling polarity. To a large extent, cytoskeletal remodeling is likely governed by the polarities of signaling molecule distributions; however, their intercorrelation is far more sophisticated. A variety of mechanisms, including G-actin treadmilling (Danuser et al. 2013), dendritic nucleation at the leading edge (Mogilner and Edelstein-Keshet 2002), and hydrodynamic flow (Niwayama et al. 2011), may function together to regulate the dynamic patterns of actin-myosin flow. Future models may involve the related molecular components in cytoskeletal remodeling based on the experimental results of disrupting actin-binding proteins.

Finally, as an old saying goes, every coin has two sides; every numerical simulation platform has its advantages and limitations. In the current framework of the LBP method, combining the auto-parallelization feature of the LB method, treating the cell edge as a collection of particles, and introducing a constriction area allow us to treat the signaling cascades very efficiently and with high numerical stability. Accordingly, in the future, the LBP-based system may be further extended to a multi-cellular system, wherein the chemical interactions between cells and intracellular dynamics are of great interest. However, application of the LBP method comes at the price of losing mechanical details. Currently, problems such as the stochastic features of cell edge protrusions-contractions, the effects of cell shape feedback on cell motility, and the interactions of cells with obstacles have not been included. To these regards, the cellular Potts method (Marée et al. 2012) is more ideal.

Acknowledgements We thank Chunfang Tong, Pan Guo, and Wenhui Hu for performing the supplemental experiments of fMLP-induced chemotaxis of neutrophils to validate our modeling. This work was supported by National Natural Science Foundation of China Grants 31230027, 91539119, and 11502272, the National Key Research and Development Program of China Grant 2016YFA0501601, the Strategic Priority Research Program Grant XDB22040101, and the Frontier Science Key Project Grant QYZDJ-SSW-JSC018.

\section{Compliance with ethical standards}

Conflict of interest The authors declare that they have no conflict of interest.

\section{References}

Ayodele S, Varnik F, Raabe D (2011) Lattice Boltzmann study of pattern formation in reaction-diffusion systems. Phys Rev E 83(1):016702

Bagorda A, Parent CA (2008) Eukaryotic chemotaxis at a glance. J Cell Sci 121(16):2621-2624

Bell GI (1978) Models for the specific adhesion of cells to cells. Science 200(4342):618-627

Bell GI, Dembo M, Bongrand P (1984) Cell adhesion. Competition between nonspecific repulsion and specific bonding. Biophys $\mathrm{J}$ 45(6): 1051-1064

Besser A, Schwarz US (2007) Coupling biochemistry and mechanics in cell adhesion: a model for inhomogeneous stress fiber contraction. New J Phys 9(11):425

Block H, Stadtmann A, Riad D, Rossaint J, Sohlbach C, Germena G, Wu D, Simon SI, Ley K, Zarbock A (2016) Gnb isoforms control a signaling pathway comprising $\operatorname{Rac} 1, \operatorname{Plc} \beta 2$, and $\mathrm{Plc} \beta 3$ leading to LFA-1 activation and neutrophil arrest in vivo. Blood 127(3):314 324

Böttcher RT, Stremmel C, Meves A, Meyer H, Widmaier M, Tseng HY, Fässler R (2012) Sorting nexin 17 prevents lysosomal degradation of $\beta 1$ integrins by binding to the $\beta 1$-integrin tail. Nat Cell Biol 14(6):584

Bottino D, Mogilner A, Roberts T, Stewart M, Oster G (2002) How nematode sperm crawl. J Cell Sci 115(2):367-384

Camley BA, Zhao Y, Li B, Levine H, Rappel WJ (2013) Periodic migration in a physical model of cells on micropatterns. Phys Rev Lett 111(15): 158102

Cao X, Moeendarbary E, Isermann P, Davidson PM, Wang X, Chen MB, Burkart AK, Lammerding J, Kamm RD, Shenoy VB (2016) A chemomechanical model for nuclear morphology and stresses during cell transendothelial migration. Biophys J 111(7):15411552

Choi CK, Vicente-Manzanares M, Zareno J, Whitmore LA, Mogilner A, Horwitz AR (2008) Actin and $\alpha$-actinin orchestrate the assembly and maturation of nascent adhesions in a myosin II motorindependent manner. Nat Cell Biol 10(9):1039

Dalous J, Burghardt E, Müller-Taubenberger A, Bruckert F, Gerisch G, Bretschneider T (2008) Reversal of cell polarity and actin-myosin cytoskeleton reorganization under mechanical and chemical stimulation. Biophys J 94(3):1063-1074

Damoulakis G, Gambardella L, Rossman KL, Lawson CD, Anderson KE, Fukui Y, Welch HC, Der CJ, Stephens LR, Hawkins PT (2014) P-Rex1 directly activates RhoG to regulate GPCRdriven Rac signalling and actin polarity in neutrophils. J Cell Sci 127(11):2589-2600

Danuser G, Allard J, Mogilner A (2013) Mathematical modeling of eukaryotic cell migration: insights beyond experiments. Annu Rev Cell Dev Biol 29:501-528

Dawes AT, Edelstein-Keshet L (2007) Phosphoinositides and Rho proteins spatially regulate actin polymerization to initiate and maintain directed movement in a one-dimensional model of a motile cell. Biophys J 92(3):744-768

Diamond MS, Springer TA (1993) A subpopulation of Mac-1 (CD11b/CD18) molecules mediates neutrophil adhesion to ICAM1 and fibrinogen. A subpopulation of Mac-1 (CD11b/CD18) molecules mediates neutrophil adhesion to ICAM-1 and fibrinogen. J Cell Biol 120(2):545-556 
Dokukina IV, Gracheva ME (2010) A model of fibroblast motility on substrates with different rigidities. Biophys J 98(12):2794-2803

Elosegui-Artola A, Bazellières E, Allen MD, Andreu I, Oria R, Sunyer R, Gomm JJ, Marshall JF, Jones JL, Trepat X et al (2014) Rigidity sensing and adaptation through regulation of integrin types. Nat Mater 13(6):631

Feng S, Zhu W (2014) Bidirectional molecular transport shapes cell polarization in a two-dimensional model of eukaryotic chemotaxis. J Theor Biol 363:235-246

Feng S, Zhou L, Lü S, Zhang Y (2018) Dynamic seesaw model for rapid signaling responses in eukaryotic chemotaxis. Phys Biol 15(5):056004

Fenteany G, Glogauer M (2004) Cytoskeletal remodeling in leukocyte function. Curr Opin Hematol 11(1):15-24

Gamba A, de Candia A, Di Talia S, Coniglio A, Bussolino F, Serini G (2005) Diffusion-limited phase separation in eukaryotic chemotaxis. Proc Natl Acad Sci USA 102(47):16927-16932

Gardiner EM, Pestonjamasp KN, Bohl BP, Chamberlain C, Hahn KM, Bokoch GM (2002) Spatial and temporal analysis of Rac activation during live neutrophil chemotaxis. Curr Biol 12(23):2029-2034

Gerisch G, Keller H (1981) Chemotactic reorientation of granulocytes stimulated with micropipettes containing fMet-Leu-Phe. J Cell Sci 52(1): $1-10$

Giannone G, Sheetz MP (2006) Substrate rigidity and force define form through tyrosine phosphatase and kinase pathways. Trends Cell Biol 16(4):213-213

Gracheva ME, Othmer HG (2004) A continuum model of motility in ameboid cells. Bull Math Biol 66(1):167-193

He S, Ji B (2016) Mechanics of cell mechanosensing in protrusion and retraction of lamellipodium. ACS Biomater Sci Eng 3(11):29432953

Heit B, Robbins SM, Downey CM, Guan Z, Colarusso P, Miller BJ, Jirik FR, Kubes P (2008) PTEN functions to 'prioritize' chemotactic cues and prevent 'distraction' in migrating neutrophils. Nat Immunol 9(7):743

Herant M, Heinrich V, Dembo M (2005) Mechanics of neutrophil phagocytosis: behavior of the cortical tension. J Cell Sci 118(9):1789-1797

Holmes WR, Edelstein-Keshet L (2012) A comparison of computational models for eukaryotic cell shape and motility. PLoS Comput Biol 8(12):e1002793

Holmes WR, Lin B, Levchenko A, Edelstein-Keshet L (2012) Modelling cell polarization driven by synthetic spatially graded Rac activation. PLoS Comput Biol 8(6):e1002366

Houk AR, Jilkine A, Mejean CO, Boltyanskiy R, Dufresne ER, Angenent SB, Altschuler SJ, Wu LF, Weiner OD (2012) Membrane tension maintains cell polarity by confining signals to the leading edge during neutrophil migration. Cell 148(1-2):175-188

Iglesias PA, Devreotes PN (2008) Navigating through models of chemotaxis. Curr Opin Cell Biol 20(1):35-40

Janmey PA, Miller RT (2011) Mechanisms of mechanical signaling in development and disease. J Cell Sci 124(1):9-18

Janson IA, Putnam AJ (2015) Extracellular matrix elasticity and topography: material-based cues that affect cell function via conserved mechanisms. J Biomed Mater Res Part A 103(3):1246-1258

Janssens PM, Van Haastert P (1987) Molecular basis of transmembrane signal transduction in Dictyostelium discoideum. Microbiol Rev 51(4):396

Jilkine A, Marée AF, Edelstein-Keshet L (2007) Mathematical model for spatial segregation of the Rho-family GTPases based on inhibitory crosstalk. Bull Math Biol 69(6):1943-1978

Jin T (2013) Gradient sensing during chemotaxis. Curr Opin Cell Biol 25(5):532-537

Karsenti E (2008) Self-organization in cell biology: a brief history. Nat Rev Mol Cell Biol 9(3):255
Kim MC, Kim C, Wood L, Neal D, Kamm RD, Asada HH (2012) Integrating focal adhesion dynamics, cytoskeleton remodeling, and actin motor activity for predicting cell migration on 3D curved surfaces of the extracellular matrix. Integr Biol 4(11):1386-1397

Kolaczkowska E, Kubes P (2013) Neutrophil recruitment and function in health and inflammation. Nat Rev Immunol 13(3):159

Kölsch V, Charest PG, Firtel RA (2008) The regulation of cell motility and chemotaxis by phospholipid signaling. J Cell Sci 121(5):551559

Kong D, Ji B, Dai L (2010) Stabilizing to disruptive transition of focal adhesion response to mechanical forces. J Biomech 43(13):25242529

Levine H, Kessler DA, Rappel WJ (2006) Directional sensing in eukaryotic chemotaxis: a balanced inactivation model. Proc Natl Acad Sci 103(26):9761-9766

Li Z, Dong X, Wang Z, Liu W, Deng N, Ding Y, Tang L, Hla T, Zeng $\mathrm{R}, \mathrm{Li} \mathrm{L}$ et al (2005) Regulation of PTEN by Rho small GTPases. Nat Cell Biol 7(4):399

Li N, Mao D, Lü S, Tong C, Zhang Y, Long M (2013) Distinct binding affinities of Mac-1 and LFA-1 in neutrophil activation. J Immunol 190(8):4371-4381

Liang S, Fu C, Wagner D, Guo H, Zhan D, Dong C, Long M (2008) Two-dimensional kinetics of $\beta 2$-integrin and ICAM-1 bindings between neutrophils and melanoma cells in a shear flow. Am J Physiol Cell Physiol 294(3):C743-C753

Lin B, Holmes WR, Wang CJ, Ueno T, Harwell A, Edelstein-Keshet L, Inoue T, Levchenko A (2012) Synthetic spatially graded Rac activation drives cell polarization and movement. Proc Natl Acad Sci 109(52):E3668-E3677

Lum AF, Green CE, Lee GR, Staunton DE, Simon SI (2002) Dynamic regulation of LFA-1 activation and neutrophil arrest on intercellular adhesion molecule 1 (ICAM-1) in shear flow. J Biol Chem 277(23):20660-20670

Ma L, Janetopoulos C, Yang L, Devreotes PN, Iglesias PA (2004) Two complementary, local excitation, global inhibition mechanisms acting in parallel can explain the chemoattractant-induced regulation of PI $(3,4,5)$ P3 response in Dictyostelium cells. Biophys J 87(6):3764-3774

Mackenzie J, Nolan M, Insall R (2016) Local modulation of chemoattractant concentrations by single cells: dissection using a bulksurface computational model. Interface Focus 6(5):20160036

Marée AF, Jilkine A, Dawes A, Grieneisen VA, Edelstein-Keshet L (2006) Polarization and movement of keratocytes: a multiscale modelling approach. Bull Math Biol 68(5):1169-1211

Marée AF, Grieneisen VA, Edelstein-Keshet L (2012) How cells integrate complex stimuli: the effect of feedback from phosphoinositides and cell shape on cell polarization and motility. PLoS Comput Biol 8(3):e1002402

Michaelson D, Silletti J, Murphy G, D'Eustachio P, Rush M, Philips MR (2001) Differential localization of Rho GTPases in live cells: regulation by hypervariable regions and RhoGDI binding. J Cell Biol 152(1):111-126

Mogilner A, Edelstein-Keshet L (2002) Regulation of actin dynamics in rapidly moving cells: a quantitative analysis. Biophys $\mathbf{J}$ 83(3): 1237-1258

Nagano M, Hoshino D, Koshikawa N, Akizawa T, Seiki M (2012) Turnover of focal adhesions and cancer cell migration. Int J Cell Biol 2012:310616

Neilson MP, Veltman DM, van Haastert PJ, Webb SD, Mackenzie JA, Insall RH (2011) Chemotaxis: a feedback-based computational model robustly predicts multiple aspects of real cell behaviour. PLoS Biol 9(5):e1000618

Nelson RD, Quie PG, Simmons RL (1975) Chemotaxis under agarose: a new and simple method for measuring chemotaxis and spontaneous migration of human polymorphonuclear leukocytes and monocytes. J Immunol 115(6):1650-1656 
Niwayama R, Shinohara K, Kimura A (2011) Hydrodynamic property of the cytoplasm is sufficient to mediate cytoplasmic streaming in the Caenorhabditis elegans embryo. Proc Natl Acad Sci 108(29):11900-11905

Onsum M, Rao CV (2007) A mathematical model for neutrophil gradient sensing and polarization. PLoS Comput Biol 3(3):e36

Otsuji M, Ishihara S, Kaibuchi K, Mochizuki A, Kuroda S et al (2007) A mass conserved reaction-diffusion system captures properties of cell polarity. PLoS Comput Biol 3(6):e108

Parent CA (2004) Making all the right moves: chemotaxis in neutrophils and Dictyostelium. Curr Opin Cell Biol 16(1):4-13

Pelham RJ, Wang Yl (1997) Cell locomotion and focal adhesions are regulated by substrate flexibility. Proc Natl Acad Sci 94(25):13661-13665

Peng X, Huang J, Xiong C, Fang J (2012) Cell adhesion nucleation regulated by substrate stiffness: a Monte Carlo study. J Biomech 45(1):116-122

Peyton SR, Putnam AJ (2005) Extracellular matrix rigidity governs smooth muscle cell motility in a biphasic fashion. J Cell Physiol 204(1):198-209

Pollard TD, Borisy GG (2003) Cellular motility driven by assembly and disassembly of actin filaments. Cell 112(4):453-465

Postma M, Van Haastert PJ (2001) A diffusion-translocation model for gradient sensing by chemotactic cells. Biophys J 81(3):1314-1323

Pramanik M, Iijima M, Iwadate Y, Yumura S et al (2009) PTEN is a mechanosensing signal transducer for myosin II localization in Dictyostelium cells. Genes Cells 14(7):821-834

Prass M, Jacobson K, Mogilner A, Radmacher M (2006) Direct measurement of the lamellipodial protrusive force in a migrating cell. J Cell Biol 174(6):767-772

Raftopoulou M, Hall A (2004) Cell migration: Rho GTPases lead the way. Dev Biol 265(1):23-32

Rappel WJ, Levine H (2008) Receptor noise and directional sensing in eukaryotic chemotaxis. Phys Rev Lett 100(22):228101

Ridley AJ, Schwartz MA, Burridge K, Firtel RA, Ginsberg MH, Borisy G, Parsons JT, Horwitz AR (2003) Cell migration: integrating signals from front to back. Science 302(5651):1704-1709

Roca-Cusachs P, Iskratsch T, Sheetz MP (2012) Finding the weakest link-exploring integrin-mediated mechanical molecular pathways. J Cell Sci 125(13):3025-3038

Rossier O, Octeau V, Sibarita JB, Leduc C, Tessier B, Nair D, Gatterdam $\mathrm{V}$, Destaing $\mathrm{O}$, Albiges-Rizo C, Tampé R et al (2012) Integrins $\beta$ 1 and $\beta 3$ exhibit distinct dynamic nanoscale organizations inside focal adhesions. Nat Cell Biol 14(10):1057

Rottner K, Stradal TE (2011) Actin dynamics and turnover in cell motility. Curr Opin Cell Biol 23(5):569-578

Sako Y, Hiroshima M, Pack CG, Okamoto K, Hibino K, Yamamoto A (2012) Live cell single-molecule detection in systems biology. Wiley Interdiscip Rev Syst Biol Med 4(2):183-192

Sakumura Y, Tsukada Y, Yamamoto N, Ishii S (2005) A molecular model for axon guidance based on cross talk between rho GTPases. Biophys J 89(2):812-822

Sander EE, Jean P, van Delft S, van der Kammen RA, Collard JG (1999) Rac downregulates Rho activity: reciprocal balance between both GTPases determines cellular morphology and migratory behavior. J Cell Biol 147(5):1009-1022

Sarvestani AS, Jabbari E (2009) Analysis of cell locomotion on ligand gradient substrates. Biotechnol Bioeng 103(2):424-429

Sasaki AT, Janetopoulos C, Lee S, Charest PG, Takeda K, Sundheimer LW, Meili R, Devreotes PN, Firtel RA (2007) G proteinindependent Ras/PI3K/F-actin circuit regulates basic cell motility. J Cell Biol 178(2):185-191

Scales TM, Parsons M (2011) Spatial and temporal regulation of integrin signalling during cell migration. Curr Opin Cell Biol 23(5):562568
Schaefer A, Hordijk PL (2015) Cell-stiffness-induced mechanosignaling - a key driver of leukocyte transendothelial migration. J Cell Sci 128(13):2221-2230

Schwarz US, Erdmann T, Bischofs IB (2006) Focal adhesions as mechanosensors: the two-spring model. Biosystems 83(2-3):225232

Shi C, Huang CH, Devreotes PN, Iglesias PA (2013) Interaction of motility, directional sensing, and polarity modules recreates the behaviors of chemotaxing cells. PLoS Comput Biol 9(7):e1003122

Shibata AC, Chen LH, Nagai R, Ishidate F, Chadda R, Miwa Y, Naruse K, Shirai YM, Fujiwara TK, Kusumi A (2013) Rac1 recruitment to the archipelago structure of the focal adhesion through the fluid membrane as revealed by single-molecule analysis. Cytoskeleton 70(3):161-177

Shin ME, He Y, Li D, Na S, Chowdhury F, Poh YC, Collin O, Su P, De Lanerolle P, Schwartz MA et al (2010) Spatiotemporal organization, regulation, and functions of tractions during neutrophil chemotaxis. Blood 116(17):3297-3310

Stephens L, Ellson C, Hawkins P (2002) Roles of PI3Ks in leukocyte chemotaxis and phagocytosis. Curr Opin Cell Biol 14(2):203-213

Stricker J, Aratyn-Schaus Y, Oakes PW, Gardel ML (2011) Spatiotemporal constraints on the force-dependent growth of focal adhesions. Biophys J 100(12):2883-2893

Stroka KM, Aranda-Espinoza H (2009) Neutrophils display biphasic relationship between migration and substrate stiffness. Cytoskeleton 66(6):328-341

Swaney KF, Huang CH, Devreotes PN (2010) Eukaryotic chemotaxis: a network of signaling pathways controls motility, directional sensing, and polarity. Ann Rev Biophys 39:265-289

Ueda M, Shibata T (2007) Stochastic signal processing and transduction in chemotactic response of eukaryotic cells. Biophys J 93(1):11-20

Ueda M, Sako Y, Tanaka T, Devreotes P, Yanagida T (2001) Singlemolecule analysis of chemotactic signaling in Dictyostelium cells. Science 294(5543):864-867

Van Haastert PJ, Devreotes PN (2004) Chemotaxis: signalling the way forward. Nat Rev Mol Cell Biol 5(8):626

Vanderlei B, Feng JJ, Edelstein-Keshet L (2011) A computational model of cell polarization and motility coupling mechanics and biochemistry. Multiscale Model Simul 9(4):1420-1443

Vazquez F, Matsuoka S, Sellers WR, Yanagida T, Ueda M, Devreotes PN (2006) Tumor suppressor PTEN acts through dynamic interaction with the plasma membrane. Proc Natl Acad Sci USA 103(10):3633-3638

Wang F, Herzmark P, Weiner OD, Srinivasan S, Servant G, Bourne HR (2002) Lipid products of PI (3) Ks maintain persistent cell polarity and directed motility in neutrophils. Nat Cell Biol 4(7):513

Weckmann M, Becker T, Nissen G, Pech M, Kopp MV (2017) SiMA: a simplified migration assay for analyzing neutrophil migration. Cytom Part A 91(7):675-685

Weiner OD (2002) Regulation of cell polarity during eukaryotic chemotaxis: the chemotactic compass. Curr Opin Cell Biol 14(2):196202

Weiner OD, Neilsen PO, Prestwich GD, Kirschner MW, Cantley LC, Bourne HR (2002) A PtdInsP 3-and Rho GTPase-mediated positive feedback loop regulates neutrophil polarity. Nat Cell Biol 4(7):509

Wolgemuth CW, Stajic J, Mogilner A (2011) Redundant mechanisms for stable cell locomotion revealed by minimal models. Biophys J 101(3):545-553

Wong K, Pertz O, Hahn K, Bourne H (2006) Neutrophil polarization: spatiotemporal dynamics of RhoA activity support a selforganizing mechanism. Proc Natl Acad Sci USA 103(10):3639_ 3644

Wu T, Feng JJ (2015) Modeling the mechanosensitivity of neutrophils passing through a narrow channel. Biophys J 109(11):2235-2245 
Yang H, Li N, Du Y, Tong C, Lü S, Hu J, Zhang Y, Long M (2017) Neutrophil adhesion and crawling dynamics on liver sinusoidal endothelial cells under shear flow. Exp Cell Res 351(1):91-99

Yap B, Kamm RD (2005) Mechanical deformation of neutrophils into narrow channels induces pseudopod projection and changes in biomechanical properties. J Appl Physiol 98(5):1930-1939

Yeung T, Georges PC, Flanagan LA, Marg B, Ortiz M, Funaki M, Zahir N, Ming W, Weaver V, Janmey PA (2005) Effects of substrate stiffness on cell morphology, cytoskeletal structure, and adhesion. Cytoskeleton 60(1):24-34

Zaman MH, Trapani LM, Sieminski AL, MacKellar D, Gong H, Kamm RD, Wells A, Lauffenburger DA, Matsudaira P (2006) Migration of tumor cells in 3D matrices is governed by matrix stiffness along with cell-matrix adhesion and proteolysis. Proc Natl Acad Sci 103(29):10889-10894
Zhang B, Zheng Y (1998) Regulation of RhoA GTP hydrolysis by the GTPase-activating proteins p190, p50RhoGAP, Bcr, and 3BP-1. Biochemistry 37(15):5249-5257

Zhelev DV, Needham D, Hochmuth RM (1994) A novel micropipet method for measuring the bending modulus of vesicle membranes. Biophys J 67(2):720-727

Publisher's Note Springer Nature remains neutral with regard to jurisdictional claims in published maps and institutional affiliations. 